\title{
Efficient Mixed Clubs: Nonlinear-Pricing Equilibria with Entrepreneurial Managers*
}

\author{
Hideo Konishi ${ }^{\dagger}$
}

\begin{abstract}
Scotchmer and Wooders (1987) show that efficient clubs are homogeneous when consumers are divisible in Berglas's (1976) anonymous crowding model. However, if consumers are not divisible or if clubs have multiple facilities with economies of scope, mixed clubs are efficient. In such a model, we consider clubs with multiple membership policies for different types of consumers, and show the existence and efficiency of equilibrium with nonlinear policies. We employ entrepreneurial equilibrium, an equilibrium concept with profit-seeking entrepreneurs. Our theorem can be regarded as showing the existence of a core allocation that satisfies envy-free property in the absence of nonanonymous crowding effects.
\end{abstract}

Keywords: club goods, mixed club, efficiency, equilibrium, entrepreneurship, nonlinear contract, envy-free, core

JEL code numbers: C62, D51, D62, H70

${ }^{*}$ This is the text for the 2009 JEA-Nakahara Prize lecture, delivered at the Fall meeting of the Japanese Economic Association at Senshu University on 10-11 October 2009. I am grateful to Samson Alva, Robin Boadway, and an anonymous referee for their comments. Special thanks are due to Marcus Berliant and his students. Part of this paper was written when I was visiting Kyoto University (KIER and Department of Economics). I thank them for their hospitality and financial support.

${ }^{\dagger}$ Department of Economics, Boston College, USA. (E-mail) hideo.konishi@bc.edu 


\section{Introduction}

In his well-known paper, Buchanan (1965) suggested that a club organization can supply a good or service efficiently whenever a consumer's utility derived by sharing the good or service with others in a group depends on the size of the group. ${ }^{1}$ In a model in which a consumer's utility is affected by her and others' intensity of use of facilities, Berglas (1976) showed that when consumers are homogeneous, an equal-treatment efficient allocation can be supported by a single price per unit usage if congestion is anonymous. Although his proposition does not extends directly to the case of heterogeneous consumers, Berglas and Pines (1981) illustrated that if there is a complete price system based on the intensity of usage, an efficient segregation of consumers occurs in a competitive equilibrium. In a more general model, Scotchmer and Wooders (1987) proved that mixed clubs are not efficient, and that a competitive equilibrium with a complete price system is perfectly segregating and efficient (the first welfare theorem). Moreover, Scotchmer and Wooders (1987) noted that this complete price system is anonymous (a type-independent price system).

Although these results are nice, they rely on the perfect divisibility of consumers in clubs: that is, they assume there is a continuum of consumers in each club. As will be shown in the next section, if the population of each club is finite, then (i) single-price per use cannot support efficient allocations, and (ii) efficient clubs may contain multiple types. The welfare analysis in Scotchmer and Wooders (1987) essentially relies on the homogeneity of each club, so their analysis cannot be extended directly to a mixed club case. One may claim that the assumption of a continuum of consumers in each club is a good approximation. However, there is another reason to consider mixed clubs. Imagine a sports club with multiple types of facilities. There may be consumers who only want to swim, and others who only want to use treadmills. Still, in the presence of economies of scope, it may not be a good idea to create a club with only swimming pools or only treadmills. It can be more cost saving to admit both types of consumers by providing both swimming pools and treadmills only with differentiated membership policies for the two types. ${ }^{2}$ Thus, it is important to allow mixed clubs even

\footnotetext{
${ }^{1}$ See Cornes and Sandler (1996) for an overview of the theory of clubs.

${ }^{2}$ Efficiency of mixed clubs in such situations is mentioned in Berglas and Pines (1981), but not analyzed. Sandler and Tschirhart (1980) mention efficiency of multiproduct clubs with cross subsidizations.
} 
if congestion effects are anonymous. ${ }^{3}$

In the literature on local public goods, Tiebout (1956) suggested that consumers' voting with feet (self-selection) over jurisdictions achieves efficient sorting allocations. ${ }^{4}$ Ellickson, Grodahl, Scotchmer, and Zame (1999) and Allouch, Conley, and Wooders (2009) considered idealized versions of club (local public goods) economies: each club has a finite membership, while there is a continuum of clubs. They proved the existence and efficiency of equilibrium in club (or local public goods) economies under general sets of assumptions. ${ }^{5}$ These papers allow mixed clubs. However, they assume nonanonymous congestion effects (differential crowding): consumers care about which (observable) types of consumers she shares the facility with (see Wooders 1999 and Scotchmer 2002 for nice surveys of the subsequent literature). On the other hand, intensity of use by members is dropped from these models: consumers care about facilities and the membership profiles of observable types, but not about the intensity of use of the facilities. In such settings, it is natural to have efficient mixed clubs, and imposing discriminating membership fees (based on observable types) is sufficient to achieve efficient outcomes. This result is intuitive: for example, in a social dance club, the gender composition of the members matters. In contrast with this line of the literature, this paper follows the approach of Berglas's and Scotchmer-Wooders's anonymous crowding model with intensity of use, taking the adverse-selection problem seriously.

The results of this paper are as follows. We first show that if members are indivisible, then efficient clubs are in general mixed even with anonymous crowding and without the economies of scope of Berglas's (1976) intensity

\footnotetext{
${ }^{3}$ In the subsequent literature, the authors dropped intensity of use from each consumer's choice variable, and assumed that consumers care about the number of members $t$ (anonymous congestion case) or the membership composition of (observable) types (nonanonymous congestion case).

${ }^{4}$ Rosen (1974) proposed a hedonic price model in which there exists a market for any commodities with any characteristics. Rosen's theory has been used in empirical and theoretical lierature. This huge literature is also based on Tiebout's original idea.

${ }^{5}$ Ellickson et al. (1999) allow consumers belong to multiple clubs and there can be a continuum of types of consumers, while the number of types of clubs is finite. Allouch et al. (2009) assume that consumers belong to a jurisdiction only and the number of consumer types is finite, while there is a continuum of types of jurisdictions. In this paper, we assume most restrictive case of the above two lines of research, while we allow intensity of use and multiple policies are provided in the same club to allow differential treatment to them.
} 
of use model. We then show that although two-part tariff policies work to support the optimal allocations when consumers are homogeneous, they do not do a good job when there are heterogeneous consumer types: the adverse selection problem makes it impossible to support an efficient allocation by two-part tariff policies. With this in mind, and allowing for economies of scope in club facility provisions, we develop a general model of a market for clubs with anonymous crowding effects. We prove the existence and effciency of an appropriately defined equilibrium assuming entrepreneurial club managers, when these club managers have a rich membership policy space that includes highly nonlinear membership policies. ${ }^{6}$ This approach is particularly interesting, since we can support efficient mixed-club allocations without discriminatory membership policies. It is natural to assume that club managers cannot discriminate consumers by their preference types, and this creates an interesting adverse selection problem.

When each club offers multiple policies (each policy is a function of intensity of use), consumers self-select their favorite club-policy pairs. Note that, in general, consumers play a game in choosing their intensity of use of the facilities in their clubs. Thus, it is important that they have beliefs about what equilibria would be realized in each potential club (nonexisting clubs in equilibrium). Following Zame (2007), we define equilibrium assuming that consumers have common beliefs about what allocation would be realized if a nonexisting (potential) club is created (common belief equilibrium). However, when highly nonlinear policies are available, we can effectively pin down each member's intensity of use. With this device, we can make the equilibrium allocation belief-free in the sense that managers can pin down the intraclub allocations that would be realized under their choices of policies.

An additional point that differentiates this paper from the existing literature is that we do not assume a complete price system for every potential club type: we assign membership prices only to existing club types. Most papers in the literature assume that the market system is complete: for every potential club type, there are certain membership prices. Managers and consumers are price-takers, and a nonexisting club in equilibrium is just a result

\footnotetext{
${ }^{6}$ Helsley and Strange (1991) examined a competitive provision of club goods with costly policy provision (provision costs differ depending on the complexity of policy types). Assuming that consumers are homogeneous, they show that the competitive equilibrium provision of club goods with endogenously chosen policy type is constrained Pareto efficient. Their "competitive" equilibrium concept appears to involve entrepreneurial managers (see below).
} 
of nobody wanting to join that club type and no manager wanting to supply that club type. Instead, assuming that club managers are profit-seeking entrepreneurs and that they have rich policies available, we manage to prove the first welfare theorem. This equilibrium concept was proposed by Konishi (2008) in a similar economy with land and other spatial elements in the spirit of Rothschild and Stiglitz (1976), ${ }^{7}$ but the proof of the existence and efficiency of equilibrium in Konishi (2008) is completely dependent on homogeneous clubs via perfect segregation, and is not applicable to the current problem.

In order to prove the existence of entrepreneurial equilibrium with mixed clubs, we will take an indirect path. We first define a social equilibrium, which is an envy-free market equilibrium, and show its existence. Then, we prove that every social equilibrium is an entrepreneurial equilibrium. In order to prove the existence of a social equilibrium, we will assign an efficient allocation to every potential type of mixed clubs. Unlike in other papers, we need convex preferences in intraclub intensity of use in order to assign a Pareto-efficient allocation in each potential club by applying the fixed point mapping developed by Shafer and Sonnenschein (1975).

Kaneko and Wooders (1986) considered a nontransferable utility game with a finite type and atomless players by assuming that coalitions can have only finite populations. They proved the nonemptiness of the equaltreatment core. Allouch et al. (2008) applied this theorem to prove the existence of equilibrium in their economy. However, we cannot apply this method directly to our problem. It is because we need to show that there is a core allocation with envy-freeness. We need to stick to an economic environment for this reason. Otherwise, some type of consumers will not want to fill the membership seats that they are supposed to fill: instead, they may want to take some other membership seats prepared for other types. Our theorem can be regarded as the existence theorem for an envy-free core allocation in our club economy.

Recently, Zame (2007) considered a general equilibrium model of firms with adverse selections and moral hazard by extending the approach of Ellickson et al. (1999). He showed the existence of equilibrium and provided examples of economies that have Pareto-ordered equilibria (thus, the first

\footnotetext{
${ }^{7}$ Wooders (1978) and Bewley (1981) also proposed equilibrium concepts in which only existing clubs are priced. Their equilibria achieve efficiency through potential coalitional deviations by consumers (instead of managers' entreneurialship).
} 
welfare theorem does not hold). Our model is the closest to this one. However, our club model has a special property: due to anonymous crowding effects, neither club managers nor club members care about the (preference) type profiles of the members. As long as the chosen actions (a vector of intensity of use) are the same, they do not care about the rest. This is precisely why we can prove the first welfare theorem in our club economy, unlike Zame (2007).

This paper is organized as follows. Section 2 reviews Berglas's analysis (1976), and shows that even in a simple and well-behaved economy, efficient clubs may require a mixed population, and that two-part tariff policies may not be able to support an efficient allocation, even if the efficient allocation consists of only homogeneous clubs. In Section 3, we provide a general model and define entrepreneurial equilibrium. Section 4 proves the existence and efficiency of entrepreneurial equilibrium when managers can choose their policies from a rich policy set. Section 5 concludes.

\section{The Berglas Model}

\subsection{Perfectly divisible members}

Although Buchanan (1965) is widely recognized as the first paper in the literature of the theory of clubs, the original idea of this general field is provided by Tiebout (1956). In the theory of clubs, Berglas (1976) made an interesting point: under certain conditions, a simple market mechanism achieves the first best allocation. In the following, we will describe a simplified version of the model by Berglas (1976). There are two types of goods: a numeraire and a club good (a facility in a club). A consumer can join at most one club, and can use a unique facility with intensity $v \in \mathbb{R}_{+}$(hours of utilization). There are identical consumers whose utility is (if she belongs to a club and utilizes the facility with intensity $v$ )

$$
x+u(v, H v),
$$

where $x \in \mathbb{R}_{+}$is the numeraire consumption and $H$ is the number (measure) of members in the club. Subutility function $u$ is increasing in the first argument (personal intensity of use) and is decreasing in the second (the aggregated intensity of use: congestion). Here, we are assuming that all members utilize the facility with the same frequency $v$. We focus on symmertic 
allocations. Each consumer is endowed with $\omega>0$ units of the numeraire. Providing a club with the facility is costly, and it costs $c>0 .{ }^{8}$

There are two possible assumptions on $H$. One is $H \in \mathbb{R}_{+}$, and the other is $H \in \mathbb{Z}_{++}$. The latter is a closer approximation to a small club, but the former may not be bad for a large club. Berglas and others in the literature assume the former, and that utility function is differentiable with all arguments.

Now, we consider a benevolent club planner's utility-maximization problem of its members given the club's resource constraints. The planner can choose the best number of members in order to maximize the representative member's utility. This treatment may sound odd, but if a club achieves this utility level, all consumers will want to join in replicas of such a club, and all other types of club will die out. So, if such an optimal club can be supported by a price system, we can say that there is a Pareto-efficient equilibrium in a club economy (though this does not say anything about whether all equilibria are efficient). To illustrate Berglas's result, fixing a facility provided by a club,${ }^{9}$ we set up the following problem (quasi-linearity is not needed for his result: it is assumed for simplicity):

$$
\max _{x, v, H} x+u(v, H v) \text { subject to } \omega=x+\frac{c}{H},
$$

where $\omega, x, v$ and $V$ are private good endowment and consumption, individual and aggregate intensity of use of the club's facility, respectively, $u(v, V)$ is a subutility function, and $H$ is the number (measure) of members. The first-order conditions are:

$$
\begin{gathered}
u_{v}+H^{*} u_{V}=0, \\
v^{*} u_{V}=-\frac{c}{\left(H^{*}\right)^{2}} .
\end{gathered}
$$

By rewriting the second equation, we have

$$
H^{*} u_{V}=-\frac{c}{H^{*} v^{*}}
$$

\footnotetext{
${ }^{8}$ Berglas's theory works in a more general setting: it is not necessary that the utility function be quasi-linear. Club service provision cost can be a function $C(H v)$. We adopted simplifying assumptions for expositional purpose.

${ }^{9}$ If the size of club facility can be selected from the real line, Boadway (1980) showed that Berglas's result holds only if clubs earn zero profits in equilibrium. In the present paper, this issue is avoided by assuming that there are only (finite) discrete public facilities by employing the public project approach introduced in Mas-Colell (1980).
} 
Putting these two equations together, we obtain

$$
u_{v}=\frac{c}{H^{*} v^{*}} .
$$

This is an interesting condition. If the consumer is myopic (i.e., ignores the effect that her consumption of facility $v$ does not affect the third term $H v$ ), by setting user price $q^{*}=u_{v}$, we can support this optimal allocation. This can be seen as follows.

The total revenue the club obtains is

$$
q^{*} \times H^{*} v^{*}=c .
$$

This immediately implies that the club's budget is balanced. Note that

$$
u_{2}=-H^{*} u_{V}
$$

shows that user price $q$ includes a Pigouvian tax to correct the negative externalities.

Proposition 0. (Berglas 1976; Berglas and Pines 1981) If the population is perfectly divisible (and differentiable), and if consumers are myopic, then an optimal club can be supported by price $q^{*}=\frac{c}{H^{*} v^{*}}$.

This is a very nice result, since it basically says that the market solves all problems even though there are intraclub externalities. However, we can make a few observations. First, does a consumer ignore a change in aggregated usage frequency caused by his/her own utilization of a facility? If there is only a finite number of consumers, in a Nash equilibrium setup with a finite population, each consumer tries to equate $u_{v}+u_{V}$ with $q^{*}$ (since she takes other consumers' consumption $(H-1) v$ as given in choosing her own $v$ ). This reduces her intensity of use. As a result, a budget shortage occurs if there is no membership fee (two-part tariff). However, obviously, if the number of members is very large, we can ignore the above point. In such a case, $H^{*} v^{*}$ is a very large number in comparison with $v^{*}$, so the effect of the third term disappears. So, with a large number of members, this issue may not be a serious problem.

Second, and more importantly, the specification of the utility function $u(v, H v, H)$ does not work. A term $\partial u / \partial H$ remains, and a budget shortage occurs. Including argument $H$ is assumed in the original club goods paper by Buchanan (1965). 
Third, Berglas assumes that there is only one type of consumer. If there are multiple types, then depending on how consumers perceive negative externalities from a high intensity of use of the facility, charged prices are different. Thus, if club managers cannot identify consumers' types (or if differentiated treatment of consumers is illegal), then the first-best allocation cannot be decentralized by such a simple market price system. In contrast, Tiebout's theory is all about voting with feet: self-selection under asymmetric information (Tiebout 1956).

Fourth, Berglas's analysis does not allow multiple types of facilities with economy of scope; if there are two types of facilities, and the cost function exhibits economies of scope $\left(c_{12}<c_{1}+c_{2}\right.$, where $c_{1}, c_{2}$, and $c_{12}$ are costs to provide facility 1 , facility 2 , and facilities 1 and 2 together, respectively), then mixed clubs are required in order to achieve efficiency even if each type of consumer cares only about one of the two facilities. ${ }^{10}$

In this paper, we take these problems seriously, and determine the conditions under which market equilibrium exists and is Pareto efficient. In the next section, we will consider the case of two-part tariff policies instead of the single-price policy considered in Berglas (1976).

\subsection{Clubs with indivisible members: the integer prob- lem}

The following example is useful in investigating the importance of integer constraints. Below, we first analyze the case without the integer problem.

Example (Gullivers and Lilliputs). Let $c=1$. Consider the following utility function:

$$
u_{t}(v, V)=t \log \frac{v}{t}-t V
$$

where $t>0$ is a taste parameter that represents a type of consumer. Now, supposing that we can choose the number for membership $H$ optimally without worrying about the integer problem, the optimization problem becomes:

$$
\max _{v, H}\left(u_{t}(v, H v)-\frac{c}{H}\right)=\max _{v, H}\left(t \log \frac{v}{t}-t H v-\frac{1}{H}\right) .
$$

\footnotetext{
${ }^{10}$ Brueckner and Lee (1991) analyze multiple club goods and economies of scope, but their point is different from ours. Their interest is whether bundling services is more efficient than separate clubs providing different services.
} 
The first-order conditions are:

$$
v: \quad t \times \frac{1}{t} \times \frac{t}{v}-t H=0,
$$

and

$$
H: \quad-t v+\frac{1}{H^{2}}=0 .
$$

These two conditions imply

$$
H^{*} v^{*}=1 \text { and } t=v^{*}=\frac{1}{H^{*}} .
$$

That is, $V^{*}=H^{*} v^{*}=1$ irrespective of the value of $t$, while the population

$H^{*}=\frac{1}{t}$ is a decreasing function in $t$. This means that consumers with a small value $t$ need a high population, but intensity of use is low. As a result, the aggregate intensity of use is still the same at the optimum. Ignoring the integer problem, the optimal allocation for type $t$ is $(v, V)=(t, 1)$ for all $t$. That is, $t$ represents the size of a consumer relative to the optimal allocation. If a Gulliver type's $t^{G}$ is twice as much as a Lilliput type's $t^{L}$, then a Gulliver is twice as big as a Lilliput, and the optimal population of the Gulliver type's club is a half of one of the Lilliput type's.

With this example, we can show the following result.

Proposition 1. With the integer problem, the optimal homogeneous club allocation can be dominated by a mixed-club allocation even if there is only one facility (no economies of scope).

Proof. Consider the above example with $t^{G}=0.07$ and $t^{L}=0.03$. In this case, neither the Gulliver type only nor the Lilliput type only can achieve $V=1$ with optimal intensity of use $v^{G}=0.07$ and $v^{L}=0.03$. However, for example, 10 Gulliver types and 10 Lilliput types together can achieve $V=1$. Hence, the optimal homogeneous club allocation is not Pareto efficient.

\subsection{Two-part tariff and intraclub game}

The goal of our analysis is to determine whether the first welfare theorem holds with two-part tariff policies. To do so, we need to know what Nash equilibria can emerge when each type of club (membership profile) is realized. We show that there is a unique Nash equilibrium under reasonable conditions 
(with quasi-linear preferences). As a corollary, we can show that in a totally segregated club, a symmetric allocation is the unique equilibrium outcome. Consider a club with $H$ consumers with a policy of $q(v)=q_{H}+q_{v} v$. In the following analysis of this section, we do not specify each consumer's type, and conduct a general analysis to explore properties of Nash equilibria. Since player $i$ is free to choose a bundle $\left(x_{i}, v_{i}\right)$ in her budget set given other players' intensity of use $V_{-i}=\sum_{j \neq i} v_{j}$. Player $i$ 's optimization problem is

$$
\max _{x_{i}, v_{i}} x_{i}+u_{i}\left(v_{i}, V_{-i}+v_{i}, H\right) \text { subject to } q_{v} v_{i}+x_{i} \leq \omega-q_{H}
$$

where $u_{i}: \mathbb{R}_{+}^{2} \times \mathbb{Z}_{++} \rightarrow \mathbb{R}$ is $C^{2}$ continuously differentiable in $\left(v_{i}, V\right)$, and $u(0, V, H)=0$ for all $V$ and $H$. The latter condition says that using facility $\left(v_{i}>0\right)$ is essential. By substituting budget constraints for $x_{i}$, the problem is written as

$$
\max _{v_{i}} u\left(v_{i}, V_{-i}+v_{i}, H\right)+\omega-q_{H}-q_{v} v
$$

The first-order condition with respect to $v_{i}$ is

$$
u_{i v}\left(v_{i}, V_{-i}+v_{i}, H\right)+u_{i V}\left(v_{i}, V_{-i}+v_{i}, H\right)=q_{v} .
$$

A Nash equilibrium is a strategy profile $\left(v_{1}, \ldots, v_{H}\right)$ such that $v_{i}$ is the optimal choice given the budget constraint and other players' intensity of use for all $i$. We can now prove the following.

Proposition 2. Suppose that the utility function is quasi-linear in the numeraire. For all $H$, and two-part tariff $q_{H}+q_{v} v$ with $q_{H}, q_{v} \geq 0$, a club with planned population $H$, there exists a unique interior Nash equilibrium in which the same consumer types choose the same $v$, if the following conditions are satisfied for all $i, v, V$, and $H$ :

1. $u_{i v}>0, u_{i V}<0, u_{i v v}<0, u_{i v V} \leq 0, u_{i V V}<0$, and $\left|u_{i v v} u_{i V V}-u_{i v V}^{2}\right|>$ 0 ,

2. $\lim _{v \rightarrow 0} u_{i v}(v, V, H)=\infty$,

3. $\lim _{v \rightarrow \infty} u_{i v}(v, V, H)=0$.

Proof. Totally differentiating the first-order condition, we have

$$
\left(u_{i v v}+u_{i V v}+u_{i v V}+u_{i V V}\right) d v_{i}+\left(u_{i v V}+u_{i V V}\right) d V_{-i}=0 .
$$


It is natural to assume $u_{i v}>0, u_{i V}<0, u_{i v v}<0, u_{i v V} \leq 0$ and $u_{i V V}<0$. If this is the case, (i) the second-order condition is met $\left(u_{i v v}+2 u_{i v V}+u_{i V V}<0\right)$, and (ii) we have

$$
\frac{d v_{i}}{d V_{-i}}=-\frac{u_{i v v}+2 u_{i v V}+u_{i V V}}{u_{i v V}+u_{i V V}}<-1
$$

Suppose that there are two distinct Nash equilibria $v=\left(v_{1}, \ldots, v_{H}\right)$ and $\tilde{v}=\left(\tilde{v}_{1}, \ldots, \tilde{v}_{H}\right)$. Then, without loss of generality, we can assume that $v_{i}>\tilde{v}_{i}$. This implies $v_{i}=b_{i}\left(V_{-i}\right)>b_{i}\left(\tilde{V}_{-i}\right)=\tilde{v}_{i}$, where $b_{i}(\cdot)$ is consumer $i$ 's bestresponse function, and $V_{-i}=\sum_{j \neq i} v_{j}$. Since the best-response function is decreasing, we have $V_{-i}<\tilde{V}_{-i}$. This implies that there is $j \neq i$ such that $v_{j}<\tilde{v}_{j}$ with $V_{-j}>\tilde{V}_{-j}$. Since the slope of best response is steeper than unity, it follows that $\tilde{V}_{-i}-V_{-i}<v_{i}-\tilde{v}_{i}$ and $V_{-j}-\tilde{V}_{-j}<\tilde{v}_{j}-v_{j}$. However, the former inequality implies $\tilde{V}>V$, while the latter implies $V>\tilde{V}$. This is a contradiction. Hence, if there is a Nash equilibrium, it must be unique. In the unique equilibrium, the same consumer types choose the same user frequency, since otherwise, by swapping the roles of the two consumers, we have multiple equilibria.

The existence of equilibrium is easy to show by assuming the Inada conditions for all $i, v, V$ and $H$ : that is, (i) $\lim _{v \rightarrow 0} u_{i v}(v, V, H)=\infty$, (ii) $\lim _{v \rightarrow \infty} u_{i v}(v, V, H)=0$, and (iii) $\lim _{v \rightarrow 0} u_{i V}(v, v, H)=0$. Condition (ii) together with $u_{i V}<0$ guarantees that there is $\bar{v}_{i H}<\infty$ such that for all $v \geq \bar{v}_{i H}, u_{i v}(v, v, H)+u_{i V}(v, v, H)<0$. This compactifies consumer $i$ 's strategy space, and we can apply Brouwer's fixed point theorem. The interior solution is guaranteed by conditions (i) and (iii).

Note that the above result holds for both homogeneous and mixed clubs. Using this, we can analyze the economy-wide efficient allocations and equilibria.

\subsection{Supporting optimal homogeneous clubs}

Proposition 1 showed that efficient clubs are in general mixed. In this subsection, we assume that efficiency is achieved in homogeneous clubs. We will show that even under this strong assumption, decentralizing an effcient allocation is not easy with simple policy tools.

Fix $\theta$, and consider a totally segregated club (only type $\theta$ consumers are its members). We focus on symmetric allocations, since Nash equilibrium is 
symmetric in this setup. Consider the following problem:

$$
\left(v^{\theta}, H^{\theta}\right) \in \max _{v, H} u(v, H v, H)-c .
$$

By using Proposition 2, we can support the allocation by price $q^{\theta}>0$, by setting

$$
u_{1}^{\theta}\left(v^{\theta}, H^{\theta} v^{\theta}, H^{\theta}\right)+u_{2}^{\theta}\left(v^{\theta}, H^{\theta} v^{\theta}, H^{\theta}\right)=q^{\theta} .
$$

Since at the optimum, $v^{\theta}>0$ by the assumptions in Proposition 1 , this is an interior symmetric Nash equilibrium. We know that $q_{v}^{\theta}>0$ holds for $H^{\theta} \geq 2$, since the optimality of $v^{\theta}$ requires

$$
u_{1}^{\theta}\left(v^{\theta}, H^{\theta} v^{\theta}, H^{\theta}\right)+H^{\theta} u_{2}^{\theta}\left(v^{\theta}, H^{\theta} v^{\theta}, H^{\theta}\right)=0 .
$$

Set

$$
q_{H}^{\theta}=\frac{c-H^{\theta} v^{\theta} q^{\theta}}{H^{\theta}}
$$

This may be positive or negative due to the integer problem and the (nonmyopic) Nash condition.

Proposition 3. Suppose that the membership of a club is fixed by $H^{\theta}$ type $\theta$ consumers. Then, under the same conditions in Proposition 1 , the symmetric optimal club allocation for type $\theta,\left(v^{\theta}, H^{\theta}\right)$, is achieved as a unique Nash equilibrium with a two-part tariff policy $\left(q_{v}^{\theta}, q_{H}^{\theta}\right)$, where

$$
u_{v}^{\theta}\left(v^{\theta}, H^{\theta} v^{\theta}, H^{\theta}\right)+u_{V}^{\theta}\left(v^{\theta}, H^{\theta} v^{\theta}, H^{\theta}\right)=q_{v}^{\theta},
$$

and

$$
q_{H}^{\theta}=\frac{1}{H^{\theta}}\left(c-H^{\theta} v^{\theta} q^{\theta}\right)
$$

Let us go back to the Gulliver-Lilliput example in Section 2.2. If $t^{G}=0.1$ and $t^{L}=0.05$, then the optimal homogeneous club allocations are $\left(v^{G}, V^{G}, H^{G}\right)=$ $(0.1,1,10)$ and $\left(v^{L}, V^{L}, H^{L}\right)=(0.05,1,20)$, respectively. These allocations are supportable by $\left(q^{G}, q_{H}^{G}\right)=(0.9,0.01)$ and $\left(q^{L}, q_{H}^{L}\right)=(0.95,0.0025)$. At the optimal allocations, consumers receive lump sum transfers and pay usage fee. An obvious corollary is the case of homogeneous type.

Corollary 1. Suppose that there is only one type of consumer. Then, the symmetric optimal club allocation is achieved as unique Nash equilibrium with a two-part tariff policy. 
What if there are multiple types? Unfortunately, we no longer have such a result even if there is an efficient allocation with homogeneous clubs.

Proposition 4. With the integer problem, even if efficient clubs are homogeneous, the optimal homogeneous club allocation may not be supportable by two-part tariff policies when there are two types.

Proof. By a variation of the Gulliver-Lilliput example. Let

$$
u^{t}(v, V)-x=t \log \frac{v}{t}-V-x .
$$

Note that coefficient $t$ of $V$ is dropped. This makes the optimal $V$ for homogeneous club be dependent on $t$. Let $V=H v$ and $x=\frac{c}{H}=\frac{1}{H}$. The first order conditions are

$$
\begin{gathered}
v: \quad t \times \frac{1}{t} \times \frac{1}{\frac{v}{t}}-H=0, \\
H: \quad-v+\frac{1}{H^{2}}=0 .
\end{gathered}
$$

As a result, the optimal allocation is described by $\left(v^{*}, H^{*}\right)=\left(t^{2}, \frac{1}{t}\right)$. Consider two types: $t_{1}=0.2$ and $t_{2}=0.1$.

For type 1 consumers, the optimal homogeneous club allocation is $\left(v_{1}^{*}, H_{1}^{*}\right)=$ $(0.04,5)$, and the optimal pricing is $q_{1 v}^{*}=\frac{t_{1}}{v_{1}^{*}}-1=4$ and $q_{1 H}^{*}=\frac{1-q_{1}^{*} v_{1}^{*}}{H_{1}^{*}}=$ $\frac{1-4 \times 0.04}{5}=0.162$. For type 2 consumers, the optimal homogeneous club allocation is $\left(v_{2}^{*}, H_{2}^{*}\right)=(0.01,10)$, and the optimal pricing is $q_{2 v}^{*}=\frac{t_{2}}{v_{2}^{*}}-1=9$ and $q_{2 H}^{*}=\frac{1-q_{2}^{*} v_{2}^{*}}{H_{2}^{*}}=\frac{1-9 \times 0.01}{10}=0.091$. These homogeneous clubs are indeed optimal, since the optimal $H^{*}$ s are integers.

Now, suppose that a type 1 consumer replaces one of 10 type 2 consumers at a type 2 optimal club. Then, the club membership is formed by one type 1 , and nine type 2 consumers. The type 1 member's intensity of use in this club will be 0.02 , and her utility will be

$$
0.2 \log \frac{0.02}{0.2}-(0.02+9 \times 0.01)-9 \times 0.02-0.091=-0.84152 .
$$

On the other hand, if she stays at a type 1 optimal club, her utility level is

$$
0.2 \log \frac{0.04}{0.2}-10 \times 0.04-4 \times 0.04-0.162=-0.84389 \text {. }
$$


Thus, the type 2 optimal clubs cannot form in equilibrium.

Thus, the two-part tariff policy does not support an efficient allocation when there are heterogeneous consumers. Note that if the type 2 optimal clubs can place an upper bound for intensity of use by each member at $\bar{v}_{2}=0.01$, the type 1 consumers do not want to join type 2 optimal clubs. This is a nonlinear price system.

In a more general model, Scotchmer and Wooders (1987) show that if clubs are homogeneous, and if there is a complete price system (a complete nonlinear pricing $q(v, H)$ for all $v$ and $H$ ), then the equilibrium is efficient. However, a complete nonlinear price system is a very complicated system. If club managers can choose facility profile $e$ as commonly assumed in the literature, then the price system must be $q(v, H, e)$. This is a hedonic price system in Rosen (1974). If mixed clubs are allowed, the situation is even worse. "Anonymous" pricing especially brings multiple complications into the model: the definition of membership policies, equilibrium concept, existence and efficiency of equilibria are all affected. In the next section, we significantly extend the model to allow mixed clubs.

\section{General Model with Nonlinear Pricing Poli- cies}

Ultimately, in order to show the existence of an efficient equilibrium, we will consider highly nonlinear policies which pin down intraclub equilibrium allocation effectively. However, we will first provide a model and equilibrium concept that allow other types of policies including two-part tariffs.

\subsection{Overview of the model}

The model assumes that there are $L$ private goods and discrete possible facility profiles. A facility profile is a list of facilities provided in a club. Facilities are categorized in $K$ groups: swimming pool, gymnasium, treadmills, exercise bikes, and rowing machines can be considered as category groups of facilities in a sports club. We assume $L$ and $K$ are finite. By abusing the notation slightly, we also denote the set of category groups by $K$. Within each category group $k \in K$, there is a set of discrete facilities $E_{k}$ ("public projects" in Mas-Colell 1980): in the swimming pool category, the size of 
the pool characterizes a facility, while in the treadmill category, the number and quality of machines characterize it. For each $E_{k}$, we have $\emptyset \in E_{k}$. The set of all possible facility profiles is denoted by $E=\Pi_{k \in K} E_{k} \cdot{ }^{11}$ A typical facility profile $e=\left(e_{1}, e_{2}, \ldots, e_{K}\right) \in \Pi_{k \in K} E_{k}$ is the list of equipment that a club can install. Each facility profile $e \in E$ can be produced by using private goods. In order to produce the facility profile $e, Y(e) \subset \mathbb{R}_{+}^{L}$ is the input requirement set. ${ }^{12}$ We assume that provision of no facility is always available for club managers: $\emptyset=(\emptyset, \ldots, \emptyset) \in E$ with $Y(\emptyset)=\mathbb{R}_{+}^{L}$ (no cost for no provision: $0 \in Y(\emptyset)$ ). This setup allows economies of scope in providing different categories of facility profiles.

There is a finite number of types of consumers. The set of all types is denoted by $\Theta$, and its representative element is $\theta \in \Theta$. A type $\theta$ consumer is endowed with private good vector $\omega^{\theta} \in \mathbb{R}_{+}^{L}$, and has a utility function $u^{\theta}: \mathbb{R}_{+}^{L} \times \mathbb{R}_{+}^{K} \times \mathbb{R}_{+}^{K} \times E \times \mathbb{Z}_{++} \rightarrow \mathbb{R}$. The arguments of $u^{\theta}(x, v, V, e, H)$ are $x \in \mathbb{R}_{+}^{L}, v \in \mathbb{R}_{+}^{K}, V \in \mathbb{R}_{+}^{K}, e \in E$, and $H \in \mathbb{Z}_{++}$are private good consumption, a vector of intensity of use (hours), a vector of aggregated intensity of use by all members of the club, facility profile, and the number of club members. Each type $\theta \in \Theta$ has a population measure $N^{\theta}>0$ with $\sum_{\theta \in \Theta} N^{\theta}=1$. We assume that clubs can effectively exclude nonmembers. Note that we assume that the physical crowding effect in facility usage by the members is anonymous; it does not depend on who utilizes the facility.

\subsection{Club policies and club types}

A club manager can decide how many memberships to sell to consumers. A policy is a function $q: \mathbb{R}_{+}^{K} \rightarrow \mathbb{R}_{+}$where $q(v)$ is the price for usage of a facility profile $\left(v \in \mathbb{R}_{+}^{K}\right.$ is a list of the number of hours a member spends in the categorized equipment in a club). The space of possible policies is described by $\mathcal{Q}$. A club can offer multiple policies targeting different types of consumers: namely, for each membership seat $h=1, \ldots, H$, a club assigns a policy $q_{h} \in \mathcal{Q}$. If a club has a single policy scheme, then all policies $q_{h}$ are the same. A policy scheme is $\eta:\{1, \ldots, H\} \rightarrow \mathcal{Q}$ such that $\eta(h)=q_{h}$ is a policy for membership $h$. In the example of a sports club, if there is only a fixed membership policy, then $q_{h}=q$ for all $h$ and $q$ is a

\footnotetext{
${ }^{11}$ If $E_{k}$ is just the number of units, it becomes the multiple public goods model found in Berglas and Pines (1981).

${ }^{12}$ For simplicity, we assume that the input requirement set is not affected by consumers' intensity of use. We can generalize following Berglas (1976) as discussed in the conclusion.
} 
constant function: $q(v)=f$. We can allow category-based fixed membership fees: for $h=1, \ldots, H_{1}, q_{h}(v)=f_{1}$ if $v_{k}>0$ only for $k=1, \ldots, K_{1}$, and $q_{h}(v)=\infty$, otherwise; and for $h=H_{1}+1, \ldots, H, q_{h}(v)=f_{2}$ if $v_{k}>0$ only for $k=K_{1}+1, \ldots, K$, and $q_{h}(v)=\infty$, otherwise. If it is a two-part tariff system, then $q_{h}=q$ for all $h=1, \ldots, H$ and $q(v)=\bar{q}+\sum_{k=1}^{K} q_{k} v_{k}$ where $\bar{q}$ and $q_{k}$ s are a membership fee and the price per use of facility $k$. Obviously, we can combine them to describe richer policies. One special type of policy is fully nonlinear: $q_{h}(v)=q_{h}$ if $v \leq \bar{v}_{h}$, and $q_{h}(v)=\infty$ otherwise. This type of policy pins down each consumer's behavior, and will be used later to achieve the first-best allocation. Thus, if we allow fully nonlinear policies, a club manager can offer a variety of policies catering to each type of consumer. However, a club manager cannot discriminate against applicants by offering different pricing schemes based on their type. A club policy scheme is a list $(e, H, \eta)$. To describe an allocation, we define an assignment of seats to consumer types: a consumer type assignment of a club is a mapping $\alpha:\{1, \ldots, H\} \rightarrow \Theta$. A population composition of a club is $\left(n^{\theta}\right)_{\theta \in \Theta}$, where $n^{\theta}=\left|\alpha^{-1}(\theta)\right|$ for all $\theta \in \Theta$. A club type is a pair of a club policy scheme and a consumer type assignment $(e, y, H, \eta, \alpha)$, where $y \in Y(e)$ is an input vector to provide facility profile $e$. With an index, a type $\gamma$ club is denoted by $(e[\gamma], y[\gamma], H[\gamma], \eta[\gamma], \alpha[\gamma])$. The population composition of type $\gamma$ club $\left(n^{\theta}[\gamma]\right)_{\theta \in \Theta}$ is found easily from $\alpha[\gamma]$. The set of all club types is denoted by $\Gamma$.

\subsection{Games played in clubs}

Consider a club type $(e, z, H, \eta, \alpha)$. Order the members of the club in an arbitrary way. The type of the $h$ th member and the policy chosen by her is denoted by $\alpha(h)=\theta_{h}$ and $q_{h}=\eta(h)$, respectively, and this type $\theta_{h}$ consumer's budget constraint is

$$
p x+q_{h}(v) \leq p \omega^{\theta_{h}}
$$

where $p \in \mathbb{R}_{+}^{L}$ is a private good price vector. Thus, her best response to other members' aggregate utilization of facility profile $V_{-h}=\sum_{h^{\prime} \neq h} v_{h^{\prime}} \in \mathbb{R}_{+}^{K}$ is

$$
\begin{gathered}
\left(x_{h}\left(V_{-h}\right), v_{h}\left(V_{-h}\right)\right) \in \arg \max _{x, v} u^{\theta_{h}}\left(x, v, v+V_{-h}, e, H\right) \\
\text { subject to } \quad p x+q_{h}(v) \leq p \omega^{\theta_{h}} .
\end{gathered}
$$


An intraclub equilibrium of this generalized game (strategy set involves a budget constraint: see Debreu 1952) in the club is $\left(x_{h}^{*}, v_{h}^{*}\right)_{h=1}^{H}$ such that for all $h=1, \ldots, H$

$$
\begin{gathered}
\left(x_{h}^{*}, v_{h}^{*}\right) \in \arg \max _{x, v} u^{\theta_{h}}\left(x, v, v+\sum_{h^{\prime} \neq h} v_{h^{\prime}}^{*}, e, H\right) \\
\text { subject to } \quad p x+q_{h}(v) \leq p \omega^{\theta_{h}} .
\end{gathered}
$$

\subsection{Allocations}

To define an allocation, we impose a few assumptions on the populations of individuals and club memberships. Some of these are adopted from papers that prove the existence and efficiency of equilibrium: Ellickson et al. (1999) and Allouch et al. (2008).

Integer Populations within Clubs (IP). The number of members of a club must be integer-valued.

Large Population (LP). There is a continuum of consumers with finite types. The measure (population) of type $\theta$ consumer is denoted by $N^{\theta}>0$ and $\sum_{\theta \in \Theta} N^{\theta}=1$.

Uniformly Bounded Population of Clubs (UB). Each club can have only a finite number of memberships, and this number is bounded from above. That is, $H \leq \bar{H}<\infty$ for all clubs and policies.

Measurement Consistency (MC). Suppose that there are Lebesgue measure $\mu^{\prime}(\gamma)$ of clubs of the same type $\gamma$ that have the same population composition $\left(n^{\theta}(\gamma)\right)_{\theta \in \Theta} \in \mathbb{Z}_{+}^{|\Theta|}$. Then, the total population of type $\theta$ consumers who belong to clubs of type $\gamma$ is $\mu^{\prime}(\gamma) \times n^{\theta}(\gamma)$ for all $\theta \in \Theta$.

Assumption IP, which was already introduced above in our definition of utility functions, is standard in the literature on local public goods economies with an endogenous number of jurisdictions. Assumption LP is also standard in order to avoid integer problems that result in the nonexistence of equilibrium. Assumption UB is key for our result. By assuming finiteness of residents in each jurisdiction together with a continuum of consumers (and 
finite types) we guarantee the dissolution of integer problems. Note that UB together with LP necessarily implies that there is a continuum of jurisdictions in the economy (Ellickson et al. 1999 and Allouch et al. 2009). Bewley (1981) made many critical comments about Tiebout's tale, but his negative results are partly the result of his not adopting IP and UB: Bewley assumed that there is a finite number of jurisdictions, and as a result, LP implies that there is a continuum of consumers in jurisdictions. UB has been formulated in various ways with various labels. However, the simplest way to state it (for our purposes) is as above. The last assumption $\mathrm{MC}$ is a technical assumption in a continuum economy. It requires that the composition of a finite population aggregates nicely to a composition of a continuum population, which is first introduced by Kaneko and Wooders (1986). We use this assumption in the proof of propositions in order to aggregate consumers' jurisdiction and consumption choices. We assume IP, LP, UB, and MC throughout the paper as in Konishi (2008).

Let $\Gamma$ be the set of all possible club types. A club allocation is a mapping $\mu: \Gamma \rightarrow \mathbb{R}_{+}$such that $\mu(\gamma)$ is the Lebesgue measure of type $\gamma$ club such that $\int_{\Gamma} n^{\theta} d \mu=N^{\theta}$ for all $\theta \in \Theta$. A consumption allocation in club type $\gamma$ is a list $(x(\gamma), v(\gamma))=\left(x_{h}(\gamma), v_{h}(\gamma)\right)_{h=1}^{\bar{H}} \in\left(\mathbb{R}_{+}^{L} \times \mathbb{R}_{+}^{K}\right)^{\bar{H}}$, where $\left(x_{h}(\gamma), v_{h}(\gamma)\right)=(0,0)$ for all $h=H[\gamma]+1, \ldots, \bar{H}(\bar{H}$ is the uniform upper bound for population of clubs). This mapping assigns a consumption vector to each type of member who is assigned to policy $\ell$ in club type $\gamma$. A consumption allocation is a mapping $(x, v): \Gamma \rightarrow\left(\mathbb{R}_{+}^{L} \times \mathbb{R}_{+}^{K}\right)^{\bar{H}}$. An input allocation is $y: \Gamma \rightarrow \mathbb{R}_{+}^{L}$ such that $y(\gamma) \in Y[e[\gamma]]$. A feasible allocation is a list of club, consumption, and input allocations $(\mu,(x, v), y)$, such that

$$
\int_{\Gamma}\left(\sum_{h=1}^{\bar{H}} x_{h}+y\right) d \mu \leq \sum_{\theta \in \Theta} N^{\theta} \omega^{\theta} .
$$

An allocation is equal treatment, for all $\theta \in \Theta$, if $\mu\left(\left\{\gamma \in \Gamma: u^{\theta}\left(x_{h}(\gamma), v_{h}(\gamma), V(\gamma), e[\gamma], H[\gamma]\right)=\bar{u}^{\theta}\right.\right.$ for all $h$ with $\left.\left.\alpha[\gamma](h)=\theta\right\}\right)=$ $N^{\theta}$ holds for some $\bar{u}^{\theta} \in \mathbb{R}$.

\subsection{Common belief system}

Let $\Gamma^{*} \subset \Gamma$ be the set of existing club types. In the entrepreneurial equilibrium defined below, we price only existing club memberships, and intraclub equilibrium allocations are observable only in existing club types. However, 
club managers and consumers need to have a system of beliefs about the situations that are not realized in the actual equilibrium allocation. There are two distinct situations. First, in order to describe consumers' free mobility, we need to imagine a situation where a type of consumer takes a membership seat that is supposed to go to another type of consumer according to the club assignment function $\alpha$. The consumer contemplating to switch her club membership needs to be able to expect what would happen if she were to switch her policy. However, if such a switch occurs, then the population assignment function of the club that she moves into changes, and the intraclub equilibrium is likely to change. Second, an entrepreneurial club manager also needs to be able to expect what will happen if she creates a new club type that is currently non-existing in an equilibrium. Whether the club can attract consumers and how profitable the new club type will be are both based on her expectation. Following Zame (2007), we assume that consumers and club managers have a common belief about what equilibrium would realize for all types of clubs. A (common) belief is a mapping $(x, v): \Gamma \rightarrow\left(\mathbb{R}_{+}^{J} \times \mathbb{R}_{+}^{K}\right)^{\bar{H}}$ such that $(x, v)(\gamma)=\left(x_{h}(\gamma), v_{h}(\gamma)\right)_{h \in\{1, \ldots, H[\gamma]\}}$. We require this common belief in the spirit of subgame perfection. Clearly, the belief must be consistent with the actual intraclub equilibrium allocations in existing club types.

\subsection{Consumer's choice among available clubs}

We now discuss consumers' club choices. Given the set of available club types $\Gamma^{*} \subset \Gamma$, suppose that a type $\theta$ consumer deviates. It is a bit tricky to define such a unilateral deviation formally, since each club provides a certain number of memberships. We assume that a consumer in a club can simply move to another club by taking over someone's seat, if she wants to. We do not consider a pairwise deviation (stability in two-sided matching literature), since it may require a chain of pairwise coordinations. Although it may appear to be a strong stability concept based on consumers' optimism, in a market economy with many clubs, it may make sense. If the consumer who is trying to take over a seat pays a small extra compensation to the incumbent member, then the incumbent may give up the seat and try to find the same deal in another club of the same type (by paying a small extra compensation). Thus, given that there are many same-type clubs in the market, this assumption may not be so far off. For example, if type $\theta$ consumer moves to a type $\gamma$ club and chooses policy $h^{\prime} \in\{1, \ldots, H[\gamma]\}$ by kicking out a member who is assigned to the club and the policy, then the 
consumer-type assignment of the club would be $\alpha_{\theta \curvearrowright h^{\prime}}[\gamma]:\{1, \ldots, H[\gamma]\} \rightarrow \Theta$ such that $\alpha_{\theta \curvearrowright h^{\prime}}[\gamma](h)=\alpha[\gamma](h)$ for all $h^{\prime} \neq h$, and $\alpha_{\theta \curvearrowright h^{\prime}}[\gamma]\left(h^{\prime}\right)=\theta$. Let us call that club type $\gamma_{\theta \curvearrowright h}=\left(e[\gamma], H[\gamma], \eta[\gamma], \alpha_{\theta \curvearrowright h}[\gamma]\right)$.

A type $\theta$ consumer's utility from choosing club $\gamma$ and its policy $h$ when $\alpha[\gamma](h)=\theta$ is

$$
U^{\theta}(\gamma, h)=u^{\theta}\left(x_{h}(\gamma), v_{h}(\gamma), \sum_{\tilde{h} \in\{1, \ldots, H[\gamma]\}} v_{\tilde{h}}(\gamma), e[\gamma], H[\gamma]\right),
$$

A type $\theta$ consumer's utility from choosing club $\gamma$ and its policy $h^{\prime}$ is

$U^{\theta}\left(\gamma_{\theta \curvearrowright h^{\prime}}, h^{\prime}\right)=u^{\theta}\left(x_{h^{\prime}}\left(\gamma_{\theta \curvearrowright h^{\prime}}\right), v_{h^{\prime}}\left(\gamma_{\theta \curvearrowright h^{\prime}}\right), \sum_{\tilde{h} \in\{1, \ldots, H[\gamma]\}} v_{\tilde{h}}\left(\gamma_{\theta \curvearrowright h^{\prime}}\right), e[\gamma], H[\gamma]\right)$,

since after this deviation, club $\gamma$ becomes club $\gamma_{\theta \curvearrowright h^{\prime}}$. For all $\theta \in \Theta$, let

$$
\Gamma_{\curvearrowright}^{\theta}\left(\Gamma^{*}\right) \equiv\left\{\left(\gamma_{\theta \curvearrowright h^{\prime}}, h^{\prime}\right) \in \Gamma \times\{1, \ldots, \bar{H}\} \mid \gamma \in \Gamma^{*} \text { and } h^{\prime} \in\{1, \ldots, H[\gamma]\}\right\} .
$$

This is the set of all club types that are generated by unilateral deviations from the set of existing clubs $\Gamma^{*}$.

\subsection{Entrepreneurial equilibrium}

Our equilibrium concept is based on an idea in Rothschild and Stiglitz (1976). ${ }^{13}$ The equilibrium concept involves three things: (i) consumers are free to choose a club type and a policy among the ones existing in the market; (ii) club managers cannot distinguish applicants' types; and (iii) club managers are entrepreneurs, and in equilibrium, there is no profit opportunity by offering a non-existing club type. Recall that a type $\gamma \in \Gamma$ club corresponds to the list of characteristics $(e[\gamma], H[\gamma], \eta[\gamma], \alpha[\gamma])$. Our equilibrium is described as follows.

\footnotetext{
${ }^{13}$ Konishi (2008) uses a similar equilibrium concept to achieve efficiency of equilibrium in a local public goods economy. However, in Konishi (2008), each jurisdiction has a homogeneous polulation by the nature of the model as in Hamilton (1975). In this paper, we deal with mixed clubs to achieve efficiency.
} 
Definition. An entrepreneurial equilibrium is a list of $\left(p^{*}, \Gamma^{*}, \mu^{*},\left(x^{*}(\gamma), v^{*}(\gamma), y^{*}(\gamma)\right)_{\gamma \in \Gamma}\right)$ such that ${ }^{14}$

1. (Equilibrium Play in Each Existing/Potential Clubs)

$$
\begin{aligned}
\left(x_{h}^{*}(\gamma), v_{h}^{*}(\gamma)\right) \in & \arg \max _{x, v} u^{\alpha[\gamma](h)}\left(x, v, \sum_{h^{\prime} \in\{1, \ldots, H[\gamma]\}} v_{h^{\prime}}(\gamma)-v_{h}^{*}(\gamma)+v, e[\gamma], H[\gamma]\right) \\
& \text { subject to } p x+\eta[\gamma](h)(v) \leq p \omega^{\alpha[\gamma](h)}
\end{aligned}
$$

for all $\gamma \in \Gamma$, and all $h \in\{1, \ldots, H[\gamma]\}$,

2. (Optimal Club Choice)

For all $\theta \in \Theta$, all $\gamma \in \Gamma^{*}$, all $h \in\{1, \ldots, H[\gamma]\}$, and all $h \in \alpha[\gamma]^{-1}(\theta)$,

$$
U^{\theta}(\gamma, h)=\max _{\left(\gamma^{\prime}, h^{\prime}\right) \in \Gamma_{\curvearrowright}^{\theta}\left(\Gamma^{*}\right)} U^{\theta}\left(\gamma^{\prime}, h^{\prime}\right),
$$

3. (Population Consistency)

For all $\theta \in \Theta, \int_{\gamma \in \Gamma^{*}} n^{\theta} d \mu^{*}=N^{\theta}$ holds,

4. (Commodity Market Clearing)

$$
\int_{\gamma \in \Gamma^{*}}\left[\sum_{h=1}^{\bar{H}}\left(\omega^{\alpha[\gamma](h)}-x_{h}^{\alpha[\gamma](h)}\right)-y^{*}\right] d \mu^{*}=0,
$$

5. (Cost Minimization)

$$
\mu^{*}\left(\left\{\gamma \in \Gamma^{*}: p^{*} y^{*} \leq p^{*} Y(e[\gamma])\right\}\right)=1
$$

6. (Club's Zero Profit Condition)

$$
\mu^{*}\left(\left\{\gamma \in \Gamma^{*}: \sum_{h=1}^{\bar{H}} \eta[\gamma](h) v_{h}^{*}(\gamma)-p^{*} y^{*}(\gamma)=0\right\}\right)=1,
$$

7. (Exhausted Profit Opportunities by Clubs)

For all $\gamma^{\prime} \in \Gamma \backslash \Gamma^{*}$, if $\sum_{h=1}^{H\left[\gamma^{\prime}\right]} \eta\left[\gamma^{\prime}\right](h)\left(v_{h}^{*}\left(\gamma^{\prime}\right)\right)-p^{*} Y\left(e\left[\gamma^{\prime}\right]\right)>0$ then such a club type is infeasible, in the sense that it cannot attract some members

\footnotetext{
${ }^{14}$ In this definition, each club assigns the same intraclub equilibrium outcome to each club type $\gamma$ (conditon 1). Obviously, we can assign different equilibrium outcomes to the same club types in general. In this sense, the proposed equilibrium concept is not the most general one. More general definitions using the "distribution approach" can be found in Hart et al. (1974), Mas-Colell (1984), or Zame (2007). However, notice that we need Pareto indifference among realized equilibrium outcomes since consumers are free to choose available policies in the market. We will assume convex preferences for our theorem; we essentially lose nothing by choosing our simpler definition.
} 
in the intraclub equilibrium that is believed to result: i.e., for all $\gamma \in \Gamma^{*}$, there exists $h \in\left\{1, \ldots, H\left[\gamma^{\prime}\right]\right\}$ such that

$$
U^{\alpha\left[\gamma^{\prime}\right](h) *}>U^{\alpha\left[\gamma^{\prime}\right](h)}\left(\gamma^{\prime}, h\right),
$$

where $U^{\theta *}$ is type $\theta^{\prime}$ 's equilibrium utility $\left(U^{\theta *}=U^{\theta}\left(\gamma, h^{\prime}\right)\right.$ for all $\gamma \in \Gamma^{*}$ and all $\left.h^{\prime} \in \alpha[\gamma]^{-1}(\theta)\right)$.

The key to the above definition is that we distinguish between $\Gamma^{*}$ (observed club types) and $\Gamma \backslash \Gamma^{*}$ (unobserved club types). First, note that it is not enough to say $\mu^{*}\left(\Gamma \backslash \Gamma^{*}\right)=0$, since as long as a type of club is available (even with zero measure), consumers may be attracted to the club type.

Second, the equilibrium concept involves beliefs held by consumers and club managers, as we have discussed above. This is so we can describe consumers' optimal policy choice and club managers' profit-maximization behaviors.

Third, we assume that club managers are entrepreneurial (Condition 7). ${ }^{15}$ Although club managers can easily observe how profitable a policy is as long as there is a club that chooses that policy, if a policy is not chosen by any club, the managers need to estimate how profitable it would be by utilizing their information on consumers' utilities (in the manner of Rothschild and Stiglitz 1976). This entrepreneurship is captured in equilibrium condition 7. In contrast, if managers are passive, there can be many inefficient equilibria if no jurisdiction chooses potentially profitable policies that go unobserved. We assume that there is no market for unobserved policies $\left(\gamma \in \Gamma \backslash \Gamma^{*}\right)$. Our condition 7 is similar to a condition in the equilibrium concept of Rothschild and Stiglitz (1976).

Note that we can impose restrictions on the set of feasible policies that clubs can choose. The set $\mathcal{Q}$ can be restricted to the set of single uniform pricing rules, two-part tariffs, or fully nonlinear pricing rules.

Before stating our result, let us define a Pareto-efficient (equal treatment) allocation. Suppose that a feasible allocation $(\mu,(x, v), y)$ is an equal

\footnotetext{
${ }^{15}$ There are papers that do not use a complete price system. Wooders (1978), Bewley (1981), and Ichiishi (1981) adopted coalitional deviations by consumers in order to achieve the efficiency of equilibrium allocation without imposing a complete price system. Instead, we use entreprenuerial managers to do the same job, who cannot distinguish consumers' types (adverse selection problem: see Konishi 2008).
} 
treatment with its utility levels $\bar{u}=\left(\bar{u}^{\theta}\right)_{\theta \in \Theta}$. Then, an equal treatment allocation $(\mu,(x, v), y)$ is Pareto efficient, unless there is a feasible allocation $\left(\mu^{\prime},\left(x^{\prime}, v^{\prime}\right), y^{\prime}\right)$ such that

$\mu^{\prime}\left(\left\{\gamma \in \Gamma: \begin{array}{c}u^{\alpha[\gamma](h)}\left(x_{h}^{\prime}(\gamma), v_{h}^{\prime}(\gamma), \sum_{h^{\prime}=1}^{H[\gamma]} v_{h^{\prime}}^{\prime}(\gamma), e[\gamma], H[\gamma]\right) \geq \bar{u}^{\alpha[\gamma](h)} \\ \forall h=1, \ldots, H[\gamma]\end{array}\right\}\right)=1$

and

$\mu^{\prime}\left(\left\{\gamma \in \Gamma: \begin{array}{c}u^{\alpha[\gamma](h)}\left(x_{h}^{\prime}(\gamma), v_{h}^{\prime}(\gamma), \sum_{h^{\prime}=1}^{H[\gamma]} v_{h^{\prime}}^{\prime}(\gamma), e[\gamma], H[\gamma]\right)>\bar{u}^{\alpha[\gamma](h)} \\ \exists h=1, \ldots, H[\gamma]\end{array}\right\}\right)>0$

That is, if a positive measure of clubs can improve members' utilities (recall that there is a finite number of members in each club) without hurting others, then an equal treatment allocation is not Pareto efficient.

\section{The Main Result}

In Section 2, we obtained negative results for two-part tariff policies. What if the class of policies available to clubs is fully nonlinear? In the extreme case, if policy $q(v)$ satisfies $q(v)=q$ if $v \leq \tilde{v}$ and $q(v)=\infty$, then the club manager can effectively pin down the members' utilization of a facility profile at one point $\tilde{v}$ (if preferences are monotonic). We refer to such a policy as a fully nonlinear policy. Even if the nonlinear pricing policy is not as strict as this one, it is still possible to pin down members' utilization of a facility profile as long as the nonlinear policy schedule stays in the lower contour set. In this section, we allow club managers to offer a set of such policies with quotas (policy 1 is available for 30 members, policy 2 is available for 20 members, etc.). The benefit of this freedom is that now a club manager can control $V$ perfectly as long as the slots are filled by its members. The main result of our paper is stated below.

Theorem 1. Suppose that all fully nonlinear policies are available for managers. There exists an entrepreneurial equilibrium and is Pareto efficient under the following assumptions:

1. For all $\theta \in \Theta$, all $e \in E \backslash\{\emptyset\}$, and all $H \leq \bar{H}, u^{\theta}(x, v, V, e, H)$ is continuous and strictly increasing in $(x, v)$ and weakly decreasing in $V$ and $H$. For all $\theta \in \Theta$, all $v$, all $V$ and all $H \leq \bar{H}, u^{\theta}(x, v, V, \emptyset, H)=$ $u^{\theta}(x, 0,0, \emptyset, 1)$, 
2. For all $\theta \in \Theta$, all $e \in E$, all $H \leq \bar{H}$, all $x \in \mathbb{R}_{+}^{L}$, all $v \in \mathbb{R}_{+}^{K}$ and all $V \in \mathbb{R}_{+}^{K}, u^{\theta}(x, v, V, e, H) \geq u^{\theta}(0, v, V, e, H)$ (essentiality of private good consumption),

3. For all $\theta \in \Theta$, all $e \in E$, and all $H \leq \bar{H}, u^{\theta}(x, v, V, e, H)$ is quasiconcave in $(x, v, V)$,

4. For all $\theta \in \Theta$, all $e \in E$, and all $H \leq \bar{H}$, there exists $\bar{v}^{\theta} \in \mathbb{R}_{+}^{K}$ such that for all $V^{\prime} \geq v^{\prime} \geq \bar{v}^{\theta}, u^{\theta}\left(x, v^{\prime}+t, V^{\prime}+t, e, H\right)<u^{\theta}\left(x, v^{\prime}, V^{\prime}, e, H\right)$ for all $t \in \mathbb{R}_{+}^{K} \backslash\{0\}$ (bounded utilization of facility profile).

5. For all $e \in E, Y[e] \subset \mathbb{R}_{+}^{L}$ is nonempty and closed.

Assumption 1 is standard. Assumption 2 is also standard when consumption set is not connected (indivisible commodities: see Mas-Colell 1977, Wooders 1978, and Ellickson 1979 for the spirit of this assumption). Assumption 3 assures that consumers' utilization of facility profile is bounded from above. Since we assume $\Theta$ is a finite set, there is a uniform upper bound for all types. Assumption 4, requiring quasi-concavity including $V$, may appear unfamiliar in this particular literature, but it is commonly assumed in finite economy. It basically requires that the marginal rate of substitution of $V_{k}$ for other goods (which is positive since $V$ are negative externalities, or bads) is decreasing in other goods consumptions. That is, as other goods consumption increases we need only a small amount of $V_{k}$ increase to keep the utility level constant. Thus, it is just a standard convex preference assumption. Given that the economy is atomless, the reader may wonder why convexity of preferences is important (indeed, neither Ellickson et al., 1999, nor Allouch et al., 2008, assumes convexity of preferences). This is assumed in order to achieve Pareto optimality in each club (finite population in each club). ${ }^{16}$ Assumption 5 needs no explanation.

The strategy to prove this theorem is as follows. First, we show that all entrepreneurial equilibria are Pareto-efficient. Second, defining a social equilibrium that is an envy-free and Pareto-efficient allocation under a marketclearing private-good price vector, we show that a social equilibrium exists

\footnotetext{
${ }^{16}$ Specifically, we use the Shafer-Sonnenschein (1975) mapping for the existence of a Pareto-efficient equilibrium. Thus, we need the union of upper contour sets to have an open graph (continuity) and be (semi) convex-valued (convex preferences).
} 
and every social equilibrium can be supported as an entrepreneurial equilibrium. In proving these results, we repeatedly use nonlinear policies that pin down each member's choice at a certain intensity of use of facility profile.

Proposition 5. An entrepreneurial equilibrium is Pareto efficient when all fully nonlinear policies are available for managers.

Proof. It follows directly from Condition 7 of the definition of entrepreneurial equilibrium. Suppose that there is a Pareto-superior feasible allocation. This means that there exists a club type (with a positive measure) such that (i) every member's utility is at least as much as the equilibrium level, (ii) there is a member whose uiltity is strictly higher than the equilibrium level, and (iii) it satisfies a budget balance (due to feasibility). Let the club type have facility $e$, input vector $y \in Y[e]$, population $H$, type assignment $\left(\theta_{h}\right)_{h=1}^{H}$, and consumption vector $\left(x_{h}, v_{h}\right)_{h=1}^{H}$ such that (i) $u^{\theta_{h}}\left(x_{h}, v_{h}, V, e, H\right)>U^{\theta_{h}^{*}}$ for all $h=1, \ldots, H$, and (ii) $p^{*} \sum_{h=1}^{H} x_{h}+p^{*} y \leq p^{*} \sum_{h=1}^{H} \omega^{\theta_{h}}$, where $V=\sum_{h^{\prime}=1}^{H} v_{h^{\prime}}$ and $U^{\theta_{h} *}$ is the equilibrium utility level for type $\theta_{h}$. Let $I_{h}=p^{*} x_{h}$ for all $h=1, \ldots, H$, and let $\tilde{U}^{\theta}(I, v, V ; p)=\max _{x} u^{\theta}(x, v, V, e, H)$ subject to $p x \leq I$. By the continuity of utility functions, there exists $\epsilon>0$ such that $\tilde{U}^{\theta_{h}}\left(I_{h}-\epsilon, v_{h}, V ; p^{*}\right)>U^{\theta_{h} *}$ for all $h=1, \ldots, H$. Thus, if a club manager proposes policy fee $q_{h}\left(v_{h}\right)=p^{*} \omega^{\theta_{h}}-I_{h}+\epsilon$ and intensity of use $v_{h}$ for all $h=1, \ldots, H$, then at least type $\theta_{h}$ consumers are attracted by the offer, and the club manager can make

$$
\begin{aligned}
\sum_{h=1}^{H} q_{h}-p^{*} y & =\sum_{h=1}^{H}\left(p^{*} \omega^{\theta_{h}}-I_{h}+\epsilon\right) \\
& =p^{*} \sum_{h=1}^{H} \omega^{\theta_{h}}-p^{*} \sum_{h=1}^{H} x_{h}+H \epsilon-p^{*} y \\
& \geq H \epsilon>0 .
\end{aligned}
$$

Since fully nonlinear policies are available, the club manager can safely implement such an intraclub allocation. Note that Condition 1 in Theorem 1 requires negative externalities in $V$ and $H$ (see Konishi, Le Breton and Weber 1997). This implies that if a consumer is happy to join a club type when all the membership seats are occupied, then she is also happy to join the club type with empty seats. This assures the absence of coordination problems, and club managers do not need to take any risk in forming a profitable potential club. This is a contradiction to the supposition. 
Note that a deviating club manager does not care about which type of consumers fill the membership seats. She only cares about someone filling all the seats available in the club. This is because of anonymity in congestion. Thus, the above deviation is sufficient to show the Pareto optimality of entrepreneurial equilibrium when fully nonlinear policies are available to managers. Note also that if fully nonlinear policies are not available, the above logic does not work, and an inefficient allocation may be supported (if profitable deviation cannot be inplemented by policy restriction or by equilibrium belief).

We construct an envy-free and Pareto-efficient allocation in the following, and then show that it is indeed an entrepreneurial equilibrium. A potential club is a pair of a facility profile and members' population composition $c \in \mathcal{C}=\left\{\left(e^{\prime}, n^{\prime}\right) \in E \times \mathbb{Z}_{+}^{\Theta}: \sum_{\theta \in \Theta} n^{\theta} \leq \bar{H}\right\}$. Since $\emptyset \in E,\left(\emptyset, n^{\prime}\right) \in \mathcal{C}$. This is a finite set. We assign a consumption vector to each type of each potential club. A symmetric consumption allocation for $c=(e[c], n[c]) \in \mathcal{C}$ is a vector $\left(x^{\theta}(c), v^{\theta}(c)\right)_{\theta \in \Theta[c]} \in\left(\mathbb{R}_{+}^{L} \times \mathbb{R}_{+}^{K}\right)^{\Theta[c]}$, where $\Theta[c]=\left\{\theta \in \Theta: n^{\theta}[c]>0\right\}$. Since consumers' utility functions are quasi-concave, it makes sense to focus on symmetric consumption allocations. We need to expand the notion of the private consumption vector due to the free mobility of consumers: a type $\theta$ consumer may want to choose a policy for type $\theta^{\prime}$ in a type $c$ club. We denote her consumption choice of private goods by $x^{\theta}\left(c, \theta^{\prime}\right)$ (intensity of use is predetermined: since she chooses the policy for $\theta^{\prime}$, her intensity of use is $\left.v^{\theta^{\prime}}(c)\right)$. Now, we define social equilibrium in the following. This equilibrium concept assumes that all possible potential clubs exist, and (somewhat socialistically) allows no flexibility of choosing intensity of use of facility profile in each club. Let $m \in \mathbb{R}_{+}^{\mathcal{C}}$ be the measures of potential clubs, and let $q \in \mathbb{R}_{+}^{\mathcal{C} \times}$ be the type-dependent membership price vector for clubs such that $q^{\theta}(c)=0$ for all $\theta \notin \Theta[c]$ (only for a notational reason).

Definition. A social equilibrium is a list of $\left(p^{*}, q^{*},\left(x^{\theta *}\left(c, \theta^{\prime}\right)\right)_{c \in \mathcal{C} ; \theta \in \Theta, \theta^{\prime} \in \Theta[c]},\left(v^{*}(c), y^{*}(c), m^{*}(c)\right)_{c \in \mathcal{C}}\right)$ such that

1. (Cost Minimization)

For all $c \in \mathcal{C}, y^{*}(c) \in \arg \min _{y \in \mathbb{R}_{+}^{L}} p^{*} Y([e])$,

2. (Club Budget Balance)

For all $c \in \mathcal{C}$ with $\left.m^{*}(c)>0, \sum_{\theta \in \Theta[c]}\right]^{\theta *}(c)=p^{*} y^{*}(c)$, 
3. (Profit Maximization)

For all $c^{\prime} \in \mathcal{C}, \sum_{\theta \in \Theta\left[c^{\prime}\right]} q^{\theta *}\left(c^{\prime}\right) \leq p^{*} y^{*}\left(c^{\prime}\right)$,

4. (Optimal Private Good Consumption for Every Policy in Every Club) For all $c \in \mathcal{C}$, all $\theta \in \Theta$, and all $\theta^{\prime} \in \Theta[c]$,

$$
x^{\theta *}\left(c, \theta^{\prime}\right) \in \arg \max u^{\theta}\left(x, v^{\theta^{\prime} *}(c), V[c], e[c], H[c]\right)
$$

subject to

$$
p^{*} x \leq p^{*} \omega^{\theta}-q^{\theta^{\prime} *}(c),
$$

5. (Intraclub Pareto Efficiency) ${ }^{17}$

For all $c \in \mathcal{C}$, there is no symmetric consumption allocation for $c$, $\left(x^{\theta}(c), v^{\theta}(c)\right)_{\theta \in \Theta[c]}$ such that

$$
u^{\theta}\left(x^{\theta}(c), v^{\theta}(c), V[c], e[c], H[c]\right)>U^{\theta *}(c),
$$

for all $\theta \in \Theta[c]$, and

$$
p^{*} \sum_{\theta^{\prime} \in \Theta} n^{\theta^{\prime}}[c] x^{\theta^{\prime}}(c) \leq p^{*} \sum_{\theta^{\prime} \in \Theta} n^{\theta^{\prime}}[c] \omega^{\theta^{\prime}}-p^{*} y^{*}
$$

6. (Optimal Club Choice)

For all $c \in \mathcal{C}$ and all $\theta \in \Theta[c]$ with $m^{*}(c)>0, U^{\theta *}(c) \geq U^{\theta *}\left(c^{\prime}, \theta^{\prime}\right)$ holds for all $c^{\prime} \in \mathcal{C}$, and all $\theta^{\prime} \in \Theta\left[c^{\prime}\right]$,

7. (Population Consistency)

For all $\theta \in \Theta, \sum_{c \in \mathcal{C}} m^{*}(c) n^{\theta}[c]=N^{\theta}$,

8. (Commodity Market Clearing)

$$
\sum_{c \in \mathcal{C}} m^{*}(c)\left[\sum_{\theta \in \Theta} n^{\theta}[c]\left(\omega^{\theta}-x^{\theta *}(c)\right)-y^{*}(c)\right]=0,
$$

where $V[c]=\sum_{\theta^{\prime} \in \Theta} n^{\theta^{\prime}}[c] v^{\theta^{\prime} *}(c), H[c]=\sum_{\theta^{\prime} \in \Theta} n^{\theta^{\prime}}[c]$, and $U^{\theta *}\left(c, \theta^{\prime}\right)=u^{\theta}\left(x^{\theta *}\left(c, \theta^{\prime}\right), v^{\theta^{\prime} *}(c), V[c], e[c], H[c]\right)$ with $U^{\theta *}(c)=U^{\theta *}(c, \theta)$.

\footnotetext{
${ }^{17}$ This condition is similar to the one in the public competitive equilibrium in Foley (1967) for a pure public goods economy. However, unlike in Foley (1967), we have multiple types of clubs including nonexisting ones in equilibrium.
} 
We have the following propositions.

Proposition 6. There exists a social equilibrium.

Using Shafer and Sonnenschein (1975) for intraclub Pareto efficiency, ${ }^{18}$ and a population mapping based on consumers' optimal location choice (Konishi 1996), we can find a social equilibrium by using Kakutani's theorem. In order to apply Shafer and Sonnenschein, we use the quasi-concavity of utility functions.

Proof. Let $\Delta \equiv\left\{(p, q) \in \mathbb{R}_{+}^{L} \times \mathbb{R}_{+}^{\mathcal{C}}: \sum_{\ell=1}^{L} p_{\ell}+\sum_{c \in \mathcal{C}} q(c)=1\right\}$, which is a price simplex. For all $c \in \mathcal{C}$, all $\theta \in \Theta$ and all $\theta^{\prime} \in \Theta[c]$, let $\beta^{\theta}\left[c, \theta^{\prime}\right]$ : $\Delta \times \mathbb{R}_{+}^{K \Theta[c]} \times \mathbb{R}_{+} \rightarrow \mathbb{R}_{+}^{L}$ is such that

$$
\beta^{\theta}\left[c, \theta^{\prime}\right]\left(p,\left(v^{\tilde{\theta}}(c)\right)_{\tilde{\theta} \in \Theta[c]}, q^{\theta^{\prime}}(c)\right) \equiv \arg \max _{x \in \mathbb{R}_{+}^{L}}\left(x, v^{\theta}(c), V[c], e[c], H[c]\right)
$$

subject to

$$
p x \leq p \omega^{\theta}-q^{\theta^{\prime}}(c) .
$$

Let $\beta: \Delta \times \mathbb{R}_{+}^{K \Sigma_{c \in \mathcal{C}} \Theta[c]} \times \mathbb{R}_{+}^{\Sigma_{c \in \mathcal{C}} \Theta[c]} \rightarrow \mathbb{R}_{+}^{L \Theta \Sigma_{c \in \mathcal{C}} \Theta[c]}$ be a product of $\beta^{\theta}\left[c, \theta^{\prime}\right] \mathrm{S}$ over $\left(\theta, c, \theta^{\prime}\right)$.

Consider Hicks's composite good for private goods. Let $I^{\theta} \in \mathbb{R}_{+}$be the income that type $\theta$ consumer at club $c$ can spend for private goods consumption. For all $\theta \in \Theta$, let $\tilde{u}^{\theta}(I, v, V, e, H ; p) \equiv \max u^{\theta}(x, v, V, e, H)$ subject to $p x \leq I$. It is easy to see $\tilde{u}^{\theta}$ is continuous in $I>0$ and $p>0$. Let

\footnotetext{
${ }^{18}$ Greenberg (1978) used the Shafer-Sonnenschein method to show the existence of a $q$ majority voting equilibrium (see Konishi 1996 for an application to the existence of voting equilibrium in local public goods economy). When $q=\infty$, the $q$-majority voting rule coincides with the Pareto-rule. Ray and Vohra (1997) were the first to directly apply the Shafer-Sonnenschein method to show the existence of a Pareto-optimal allocation.
} 
$\mathcal{P}[c]: \mathbb{R}_{+}^{(1+K) \Theta[c]} \times \Delta \rightarrow \mathbb{R}_{+}^{(1+K) \Theta[c]}$ be a Pareto mapping such that

$$
\begin{aligned}
& \mathcal{P}[c]\left(\left(I^{\theta^{\prime}}, v^{\theta^{\prime}}\right)_{\theta^{\prime} \in \Theta[c]}, p\right) \\
= & \left\{\left(\tilde{I}^{\theta^{\prime}}, \tilde{v}^{\theta^{\prime}}\right)_{\theta^{\prime} \in \Theta[c]} \in \mathbb{R}_{+}^{(L+K) \Theta[c]} \mid\right. \\
& \tilde{u}^{\theta}\left(\tilde{I}^{\theta}, \tilde{v}^{\theta}, \sum_{\theta^{\prime} \in \Theta[c]} n^{\theta^{\prime}}[c] \tilde{v}^{\theta^{\prime}}, e[c], H[c]\right) \\
> & u^{\theta}\left(I^{\theta}, v^{\theta}, \sum_{\theta^{\prime} \in \Theta} n^{\theta^{\prime}}[c] v^{\theta^{\prime}}(c), e[c], \sum_{\theta^{\prime} \in \Theta} n^{\theta^{\prime}}[c]\right) \text { for all } \theta \in \Theta \\
& \text { and } \left.\sum_{\theta \in \Theta[c]} n^{\theta}[c] \tilde{I}^{\theta} \leq p \sum_{\theta \in \Theta[c]} n^{\theta}[c] \omega^{\theta}-p y(c)\right\} .
\end{aligned}
$$

There are only a finite number of types, and a finite intersection of open sets is open (relative to the feasibility correspondence: the inequality in the last line). Thus, mapping $\mathcal{P}[c]$ has an open graph (utility function is continuous). Let the set of feasible allocations in club $c$ be $\mathcal{F}[c, p] \equiv$ $\left\{\left(\tilde{I}^{\theta^{\prime}}, \tilde{v}^{\theta^{\prime}}\right)_{\theta^{\prime} \in \Theta[c]} \in \mathbb{R}_{+}^{(L+K) \Theta[c]}: \sum_{\theta \in \Theta[c]} n^{\theta}[c] \tilde{I}^{\theta} \leq p \sum_{\theta \in \Theta[c]} n^{\theta}[c] \omega^{\theta}-p y(c)\right\}$. Let $U^{S S}\left[c ;\left(I^{\theta}, v^{\theta}\right)_{\theta \in \Theta[c]}, p\right]$ be a Shafer-Sonnenschein utility function on $\mathcal{F}[c, p]$ such that $U^{S S}\left[c ;\left(I^{\theta}, v^{\theta}\right)_{\theta \in \Theta[c]}, p\right]\left(\left(\tilde{I}^{\theta}, \tilde{v}^{\theta}\right)_{\theta \in \Theta[c]}\right)=0$ if $\left(\tilde{I}^{\theta}, \tilde{v}^{\theta}\right)_{\theta \in \Theta[c]} \notin \mathcal{P}[c]\left(\left(I^{\theta}(c), v^{\theta}(c)\right)_{\theta \in \Theta[c]}, p\right)$, and $U^{S S}\left[c ;\left(I^{\theta}, v^{\theta}\right)_{\theta \in \Theta[c]}, p\right]\left(\left(\tilde{I}^{\theta}, \tilde{v}^{\theta}\right)_{\theta \in \Theta[c]}\right)$ is the distance between $\left(\tilde{I}^{\theta}, \tilde{v}^{\theta}\right)_{\theta \in \Theta[c]}$ and the topological boundary of $\mathcal{P}[c]\left(\left(I^{\theta}(c), v^{\theta}(c)\right)_{\theta \in \Theta[c]}\right)$, otherwise (see Shafer and Sonnenschein 1975). This is a continuous function in its arguments. Let $\delta[c]: \mathbb{R}_{+}^{(1+K) \Theta[c]} \times \Delta \rightarrow \mathbb{R}_{+}^{(1+K) \Theta[c]}$ be such that

$\delta[c]\left(\left(I^{\theta}, v^{\theta}\right)_{\theta \in \Theta[c]}, p\right)=\arg \max _{\left(\tilde{I}^{\theta}, \tilde{v}^{\theta}\right)_{\theta \in \Theta[c]} \in \mathcal{F}[c, p]} U^{S S}\left[c ;\left(I^{\theta}, v^{\theta}\right)_{\theta^{\prime} \in \Theta[c]}, p\right]\left(\left(\tilde{I}^{\theta}, \tilde{v}^{\theta}\right)_{\theta \in \Theta[c]}\right)$

This mapping $\delta[c]$ is designed to find intraclub Pareto-efficient allocations as a fixed point of the mapping.

Let $\delta: \mathbb{R}_{+}^{(1+K) \Sigma_{c \in \mathcal{C}} \Theta[c]} \times \Delta \rightarrow \mathbb{R}_{+}^{(1+K) \Sigma_{c \in \mathcal{C}} \Theta[c]}$ be a product of $\delta[c]$ s. Let $\operatorname{proj}_{v} \delta: \mathbb{R}_{+}^{(1+K) \Sigma_{c \in \mathcal{C}} \Theta[c]} \times \Delta \rightarrow \mathbb{R}_{+}^{K \Sigma_{c \in \mathcal{C}} \Theta[c]}$ be a project of $\delta$ on intensity of use of the facility profile (dropping income). Let $\iota: \mathbb{R}_{+}^{L \Sigma_{c \in \mathcal{C}} \Theta[c]} \times \Delta \rightarrow \mathbb{R}_{+}$be such that $\iota^{\theta}[c]=p x^{\theta}(c)$ for all $\theta \in \Theta[c]$ and all $c \in \mathcal{C}$. Let $\tilde{\delta}: \mathbb{R}_{+}^{L \Sigma_{c \in \mathcal{C}} \Theta[c]} \times$ $\mathbb{R}_{+}^{(1+K) \Sigma_{c \in \mathcal{C}} \Theta[c]} \times \Delta \rightarrow \mathbb{R}_{+}^{(1+K) \Sigma_{c \in \mathcal{C}} \Theta[c]}$ be a product of $\operatorname{proj}_{v} \delta$ and $\iota: \operatorname{proj}_{I} \delta$ is replaced by $\iota$. 
For each type club $c \in \mathcal{C}$, let the input choice correspondence be $\chi[c]$ : $\Delta_{p} \rightarrow \mathbb{R}_{+}^{L}$ such that $\chi[c](p)=\arg \min _{y \in Y[e[c]]} p y$. Let $\chi: \Delta_{p} \rightarrow \mathbb{R}_{+}^{L \mathcal{C}}$ be the product of $\chi[c] \mathrm{s}$.

Let $M \equiv\left\{m \in \mathbb{R}_{+}^{\mathcal{C}} \mid \sum_{c \in \mathcal{C}} m(c) H[c]=1\right\}$. This is the space of club distributions that are consistent with population distribution over types. Let $\tilde{M} \equiv\left\{m \in \mathbb{R}_{+}^{\mathcal{C}} \mid \sum_{c \in \mathcal{C}} m(c) H[c]=1.1\right\}$. This set can be regarded as a "free entry condition." It is because a fraction of potential club managers must choose to provide a trivial empty club (i.e., $e=\emptyset$ ) which guarantees zero profit $(0 \in Y[\emptyset])$. This device guarantees zero profit in the fixed point of the mapping. Let

$$
U^{\theta}[c] \equiv u^{\theta}\left(x^{\theta}(c), v^{\theta}(c), \sum_{\tilde{\theta} \in \Theta[c]} n^{\tilde{\theta}}[c] v^{\tilde{\theta}}(c), e[c], H[c]\right)
$$

and

$$
U^{\theta}\left[c, \theta^{\prime}\right] \equiv u^{\theta}\left(x^{\theta}\left(c, \theta^{\prime}\right), v^{\theta^{\prime}}(c), \sum_{\tilde{\theta} \in \Theta[c]} n^{\tilde{\theta}}[c] v^{\tilde{\theta}}(c), e[c], H[c]\right) .
$$

Clearly, $U^{\theta}[c, \theta]=U^{\theta}[c]$ holds. Let $R^{\theta} \equiv\left\{r^{\theta} \in \mathbb{R}_{+}^{\Sigma_{c \in \mathcal{C}} \Theta[c]} \mid \sum_{c \in \mathcal{C}, \theta^{\prime} \in \Theta[c]} r^{\theta}\left(c, \theta^{\prime}\right)=\right.$ $\left.N^{\theta}\right\}$. Let $\rho^{\theta}: \mathbb{R}_{+}^{(L+K)\left(\Sigma_{c \in \mathcal{C}} \Theta[c]\right)} \rightarrow R^{\theta}$ be such that

$$
\begin{aligned}
& \rho^{\theta}\left(\left(x^{\theta}\left(c, \theta^{\prime}\right), v^{\theta^{\prime}}(c)\right)_{c \in \mathcal{C}, \theta^{\prime} \in \Theta[c]}\right) \\
= & \left\{r^{\theta} \in R^{\theta}: r^{\theta}\left(c, \theta^{\prime}\right)>0 \Rightarrow U^{\theta}\left[c, \theta^{\prime}\right] \geq U^{\theta}\left[c^{\prime}, \theta^{\prime \prime}\right] \text { for all } c^{\prime} \in \mathcal{C} \text { and } \theta^{\prime \prime} \in \Theta\left[c^{\prime}\right]\right\} .
\end{aligned}
$$

Let $R \equiv \Pi_{\theta \in \Theta} R^{\theta}$ and $\rho: \mathbb{R}_{+}^{(L+K)\left(\Sigma_{c \in \mathcal{C}} \Theta[c]\right) \Theta} \rightarrow R$ be such that

$$
\rho\left(\left(x^{\theta}\left(c, \theta^{\prime}\right), v^{\theta^{\prime}}(c)\right)_{c \in \mathcal{C}, \theta^{\prime} \in \Theta[c], \theta \in \Theta}\right)=\Pi_{\theta \in \Theta} \rho^{\theta}\left(\left(x^{\theta}\left(c, \theta^{\prime}\right), v^{\theta^{\prime}}(c)\right)_{c \in \mathcal{C}, \theta^{\prime} \in \Theta[c]}\right) .
$$

This is a population mapping (correspondence) that assigns each type of consumers to the clubs that offer the highest utilities. Let $Z_{r} \equiv\left\{z_{r} \in[-1,1]^{\Sigma_{c \in \mathcal{C}} \Theta[c]}: \sum_{c \in \mathcal{C}} z_{r}\left(c, \theta^{\prime}\right)=0\right\}$ Let $\zeta_{r}: \mathbb{R}_{+}^{(L+K)\left(\Sigma_{c \in \mathcal{C}} \Theta[c]\right) \Theta} \times M \rightarrow Z_{r}$ be such that

$$
\zeta_{r}(\rho, m)=\left(\sum_{\theta \in \Theta} \rho^{\theta}\left(\left(x^{\theta}\left(c, \theta^{\prime}\right), v^{\theta^{\prime}}(c)\right)_{c \in \mathcal{C}, \theta^{\prime} \in \Theta[c]}\right)-m(c) \times n^{\theta^{\prime}}[c]\right) .
$$

This is an excess demand mapping for each policy at each club. Let $\zeta_{p}$ : $\mathbb{R}_{+}^{L\left(\Sigma_{c \in \mathcal{C}} \Theta[c]\right) \Theta} \times R \times \Pi_{c \in \mathcal{C}} \mathbb{R}_{+}^{L} \rightarrow Z_{p} \subset \mathbb{R}^{L}$ be such that

$$
\begin{aligned}
& \zeta_{p}\left(\left(x^{\theta}\left(c, \theta^{\prime}\right), r^{\theta}\left(c, \theta^{\prime}\right)\right)_{\theta \in \Theta, c \in \mathcal{C}, \theta^{\prime} \in \Theta[c]},(z(c))_{c \in \mathcal{C}}\right) \\
= & \sum_{c \in \mathcal{C}}\left[\sum_{\theta^{\prime} \in \Theta[c]} \sum_{\theta \in \Theta} r^{\theta}\left(c, \theta^{\prime}\right) x^{\theta}\left(c, \theta^{\prime}\right)+y(c)\right]-\sum_{\theta \in \Theta} N^{\theta} \omega^{\theta} .
\end{aligned}
$$


The club manager's profit from providing type $c \in \mathcal{C}$ club is

$$
\pi[c](p, q(c))=\sum_{\theta \in \Theta[c]} n^{\theta}[c] q^{\theta}(c)-p y(c) .
$$

Given club managers' profit-maximization behavior, if one type of club is the most profitable, all club managers are drawn to that type. Let $\mu: \Delta \rightarrow \tilde{M}$ be such that

$$
\mu(p, q)=\left\{m \in \tilde{M} \mid m(c)>0 \Rightarrow c \in \arg \max _{c \in \mathcal{C}} \pi[c](p, q(c))\right\} .
$$

Let $\sigma: Z_{p} \times Z_{r} \rightarrow \Delta$ be such that

$$
\sigma(z)=\arg \max _{(p, q) \in \Delta} \sum_{\ell \in L} p_{\ell} z_{p \ell}+\sum_{c \in \mathcal{C}} q(c) z_{r}(c) .
$$

This is a price mapping.

Our fixed-point mapping is a product of all the above mappings $\beta, \tilde{\delta}, \chi$, $\rho, \mu, \zeta$, and $\sigma$ :

$$
\begin{aligned}
\varphi: & \mathbb{R}_{+}^{(L+K) \Sigma_{c \in \mathcal{C}} \Theta[c]} \times \mathbb{R}_{+}^{\Sigma_{c \in \mathcal{C}} \Theta[c]} \times \mathbb{R}_{+}^{L \mathcal{C}} \times R \times M \times Z_{p} \times Z_{r} \times \Delta \rightarrow \\
& \mathbb{R}_{+}^{(L+K) \Sigma_{c \in \mathcal{C}} \Theta[c]} \times \mathbb{R}_{+}^{\Sigma_{c \in \mathcal{C}} \Theta[c]} \times \mathbb{R}_{+}^{L \mathcal{C}} \times R \times M \times Z_{p} \times Z_{r} \times \Delta .
\end{aligned}
$$

A fixed point of $\varphi$,

$$
\left(x^{*}, v^{*}, I^{*}, y^{*}, m^{*}, r^{*}, z_{p}^{*}, z_{r}^{*}, p^{*}, q^{*}\right) \in \varphi\left(x^{*}, v^{*}, I^{*}, y^{*}, m^{*}, r^{*}, z_{p}^{*}, z_{r}^{*}, p^{*}, q^{*}\right),
$$

is shown to be a social equilibrium.

In order to apply Kakutani's fixed-point theorem, we need compactness of choice sets. Let us truncate each consumer's consumption set $\mathbb{R}_{+}^{L}$ by a hypercube with size $s=1,2, \ldots$ : i.e., each consumer's consumption set is $X_{s} \equiv\left\{x \in \mathbb{R}_{+}^{L}: x \leq(s, s, \ldots, s)\right\}$. Let $\mathcal{V} \equiv\left\{v \in \mathbb{R}_{+} \mid v \leq \max _{\theta \in \Theta} \bar{v}^{\theta}\right\}$ (see Assumption 3). Let $\Delta^{\epsilon} \equiv\left\{(p, q) \in \mathbb{R}_{+}^{L} \times \mathbb{R}_{+}^{\Sigma_{c \in \mathcal{C}} \Theta(c)} \mid p_{\ell} \geq \epsilon\right.$ for all $\ell=1, \ldots, L\}$. The reason that $\Delta$ is truncated is that if all private good prices are allowed to become zero, then the budget correspondence does not satisfy lower hemi-continuity (a sudden expansion to the entire consumption set). Let $Z_{p s} \equiv X_{s}-\left\{\sum_{\theta \in \Theta} N^{\theta} \omega^{\theta}\right\}$. Let $Y_{s} \equiv \Pi_{c \in \mathcal{C}}\left(Y(c) \cap[0, s]^{L}\right)$ for all $s=1,2, \ldots$ : this is a truncated input requirement set. Take $s$ large enough to guarantee $Y(c) \cap[0, s]^{L} \neq \emptyset$ for all $c \in \mathcal{C}$. For fixed $s, \epsilon \in(0,1 / L)$, the 
domain (and the range) of the fixed point mapping is nonempty, compact and convex. The standard argument shows that mappings, $\beta, \tilde{\delta}, \chi, \rho, \mu$, $\zeta$ and $\sigma$ are nonempty- and convex-valued, and have closed graphs. Thus, there is a fixed point $\left(x^{\epsilon s *}, v^{\epsilon s *}, I^{\epsilon s *}, y^{\epsilon s *}, r^{\epsilon s *}, m^{\epsilon s *}, z_{p}^{\epsilon s *}, z_{r}^{\epsilon s *}, p^{\epsilon s *}, q^{\epsilon s *}\right)$.

Now, we show that this fixed point is a social equilibrium for an $(\epsilon, s)$ truncated economy. By the construction of $\beta$, in each policy of each club, every type's consumption choice is optimal (if they choose the policy). Thus, Condition 4 is met. Now, we show that in each club $c \in \mathcal{C},\left(I^{\epsilon \epsilon \theta *}(c), v^{\epsilon s \theta *}(c)\right)_{\theta \in \Theta[c]}$ is an intraclub Pareto-efficient allocation (Condition 5). We can show that at the fixed point, $\left(I^{\epsilon s \theta *}(c), v^{\epsilon s \theta *}(c)\right)_{\theta \in \Theta[c]} \in \delta[c]\left(\left(I^{\epsilon s \theta^{\prime} *}(c), v^{\epsilon s \theta^{*} *}(c)\right)_{\theta^{\prime} \in \Theta[c]}, p^{\epsilon s *}\right)$. We have the following key lemma.

Lemma 1. At the fixed point of the mapping $\varphi$ with truncated domain/range, for all $c \in \mathcal{C},\left(I^{\epsilon s \theta^{\prime} *}(c), v^{\epsilon s \theta^{\prime} *}(c)\right)_{\theta^{\prime} \in \Theta[c]}$ is an intraclub Pareto-efficient consumption vector.

Proof of Lemma 1. Suppose that $\left(I^{\epsilon s \theta^{\prime} *}(c), v^{\epsilon s \theta^{\prime} *}(c)\right)_{\theta^{\prime} \in \Theta[c]}$ is not intraclub Pareto efficient. Then, $\mathcal{P}[c]\left(\left(I^{\epsilon s \theta^{\prime} *}(c), v^{\epsilon s \theta^{\prime} *}(c)\right)_{\theta^{\prime} \in \Theta[c]}\right) \neq \emptyset$ holds. By the construction of the Shafer-Sonnenschein mapping $\delta,\left(\tilde{I}^{\epsilon s \theta^{\prime}}(c), \tilde{v}^{\epsilon s \theta^{\prime}}(c)\right)_{\theta^{\prime} \in \Theta[c]} \in$ $\delta[c]\left(\left(I^{\epsilon s \theta^{\prime} *}(c), v^{\epsilon s \theta^{\prime} *}(c)\right)_{\theta^{\prime} \in \Theta[c]}\right)$ satisfies $\left(\tilde{I}^{\epsilon s \theta^{\prime}}(c), \tilde{v}^{\epsilon s \theta^{\prime}}(c)\right)_{\theta^{\prime} \in \Theta[c]} \neq\left(I^{\epsilon s \theta^{\prime} *}(c), v^{\epsilon s \theta^{\prime} *}(c)\right)_{\theta^{\prime} \in \Theta[c]}$. However, since $\left(v^{\epsilon s \theta^{\prime} *}(c)\right)_{\theta^{\prime} \in \Theta[c]} \in \operatorname{proj}_{v} \delta[c]\left(\left(I^{\epsilon s \theta^{\prime} *}(c), v^{\epsilon s \theta^{\prime} *}(c)\right)_{\theta^{\prime} \in \Theta[c]}, p^{\epsilon s *}\right), \tilde{v}^{\epsilon s \theta^{\prime}}(c)=$

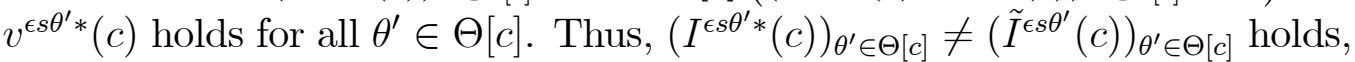
and we have

$$
\begin{aligned}
& \left.\tilde{u}^{\theta}\left(\tilde{I}^{\epsilon s \theta}(c), v^{\epsilon s \theta *}(c), \sum_{\theta^{\prime} \in \Theta} n^{\theta^{\prime}}[c] v^{\epsilon s \theta *}(c), e[c], \sum_{\theta^{\prime} \in \Theta} n^{\theta^{\prime}}[c]\right) ; p^{\epsilon s *}\right) \\
> & \left.\tilde{u}^{\theta}\left(I^{\epsilon s \theta *}(c), v^{\epsilon s \theta *}(c), \sum_{\theta^{\prime} \in \Theta} n^{\theta^{\prime}}[c] v^{\epsilon s \theta^{\prime} *}(c), e[c], \sum_{\theta^{\prime} \in \Theta} n^{\theta^{\prime}}[c]\right) ; p^{\epsilon s *}\right)
\end{aligned}
$$

for all $\theta \in \Theta[c]$. However, by mapping $\mathcal{P}[c]$,

$$
\sum_{\theta \in \Theta[c]} n^{\theta}[c] \tilde{I}^{\epsilon s \theta} \leq p^{\epsilon s *} \sum_{\theta \in \Theta[c]} n^{\theta}[c] \omega^{\theta}-p^{\epsilon s *} y^{\epsilon s *}(c) .
$$

But as we will see below, mapping $\zeta$ assures that in the fixed point, clubs get zero profits. That is,

$$
\sum_{\theta \in \Theta[c]} n^{\theta}[c] q^{\epsilon s \theta *}(c)=p^{\epsilon s *} y^{\epsilon s *}(c) .
$$


Since $\beta$ mapping shows that $x^{\epsilon s \theta *}(c)$ maximizes utility subject to $p^{\epsilon s *} x^{\theta} \leq$ $p^{\epsilon s *} \omega^{\theta}-q^{\epsilon s \theta *}(c)$, we have $p^{\epsilon s *} x^{\theta}=p^{\epsilon s *} \omega^{\theta}-q^{\epsilon s \theta *}(c)$ (strict monotonicity of preferences). Since by mapping $\iota$, we know that $I^{\epsilon s \theta *}(c)=p^{\epsilon s *} x^{\epsilon s \theta *}(c)$, we now conclude

$$
\sum_{\theta \in \Theta[c]} n^{\theta}[c] \tilde{I}^{\epsilon s \theta} \leq \sum_{\theta \in \Theta[c]} n^{\theta}[c] I^{\epsilon s \theta *}
$$

However, by mapping $\beta$ again, each type needs a higher income than the fixed-point income, $\tilde{I}^{\epsilon s \theta}>I^{\epsilon s \theta *}$. This is a contradiction. Hence, $\left(I^{\epsilon s \theta^{\prime} *}(c), v^{\epsilon s \theta^{\prime} *}(c)\right)_{\theta^{\prime} \in \Theta[c]}$ is intraclub Pareto efficient.

It is important to notice that allocation $\left(I^{\epsilon s \theta^{\prime} *}(c), v^{\epsilon s \theta^{\prime} *}(c)\right)_{\theta^{\prime} \in \Theta[c]}$ is intraclub Pareto efficient only when the policy for $\theta^{\prime}$ is actually chosen by type $\theta^{\prime}$ consumers for all $\theta^{\prime} \in \Theta[c]$. If we look at the fixed-point mapping, it is not obvious that the population composition at type $c$ club is indeed $\left(n^{\theta}[c]\right)_{\theta \in \Theta[c]}$ (it is possible that other type consumers happily chose club $c$ 's policies, and the market for club membership cleared). However, we have the following result.

Lemma 2. In the fixed point of $\varphi$, suppose that $n^{\theta}[c] m^{\epsilon s *}(c) \neq \rho_{\left(c, \theta^{\prime}\right)}^{\theta \epsilon s *}$ for some $\left(c, \theta^{\prime}\right)$ and $\theta$. Then, the allocation achieved in type $c$ club with population composition $n^{\prime} \neq n[c]$ in the fixed point is intraclub Pareto efficient in club type $c^{\prime}=\left(e[c], n^{\prime}\right) \in \mathcal{C}$.

Proof of Lemma 2. Consider a club of type $c$ with population composition $n^{\prime}$. Let $a:\{1, \ldots, H[c]\} \rightarrow \Theta\left[c^{\prime}\right] \times \Theta[c]$. That is, $a_{1}(h)=\theta$ represents $h$ 's type, and $a_{2}(h)=\theta^{\prime}$ represents $h$ 's choice of policy (catering to type $\theta^{\prime}$ consumers' preferences) in club type $c$. Thus, consumption vector $\left(x_{h}, v_{h}\right)_{h=1}^{H[c]}$ is such that $\left(x_{h}, v_{h}\right)=\left(x^{\theta \epsilon s *}\left(c, \theta^{\prime}\right), v^{\theta^{\prime} \epsilon s *}(c)\right)$ for $a_{1}(h)=\theta$ and $a_{2}(h)=\theta^{\prime}$. Let

$$
\left(x^{\theta}\left(c^{\prime}\right), v^{\theta}\left(c^{\prime}\right)\right)_{\theta \in \Theta\left[c^{\prime}\right]} \equiv\left(\frac{1}{n^{\prime \theta}[c]} \sum_{h \in\left\{h^{\prime} \mid a_{1}\left(h^{\prime}\right)=\theta\right\}} x_{h}, \frac{1}{n^{\prime \theta}[c]} \sum_{h \in\left\{h^{\prime} \mid a_{1}\left(h^{\prime}\right)=\theta\right\}} v_{h}\right)_{\theta \in \Theta\left[c^{\prime}\right]} .
$$

Note that in the fixed point, each type of consumer's policy choice is optimal: i.e., for all $h \in\left\{h^{\prime} \mid a_{1}\left(h^{\prime}\right)=\theta\right\}, u^{\theta}\left(x_{h}, v_{h}, \sum_{h^{\prime}=1}^{H[c]} v_{h^{\prime}}, e[c], H[c]\right)=$ $\max U^{\theta *}\left[c, \theta^{\prime}\right]$. Thus, we have

$$
u^{\theta}\left(x^{\theta}\left(c^{\prime}\right), v^{\theta}\left(c^{\prime}\right), \sum_{h^{\prime}=1}^{H[c]} v_{h^{\prime}}, e[c], H[c]\right) \geq \max U^{\theta *}\left[c, \theta^{\prime}\right]
$$


by the quasi-concavity of utility function.

Now, in the fixed point, in club $c^{\prime}$, an intraclub efficient allocation is chosen. Suppose that $m^{\epsilon s *}\left(c^{\prime}\right)>0$. Then, for $\theta \in \Theta\left[c^{\prime}\right]$, we have

$$
u^{\theta}\left(x^{\theta \epsilon s *}\left(c, \theta^{\prime}\right), v^{\theta \epsilon s *}(c), \sum_{\theta^{\prime} \in \Theta\left[c^{\prime}\right]} v^{\theta^{\prime} \epsilon s *}(c), e[c], H[c]\right)=\max U^{\theta *}\left[c, \theta^{\prime}\right] .
$$

Since the intraclub allocation is Pareto efficient,

$$
u^{\theta}\left(x^{\theta}\left(c^{\prime}\right), v^{\theta}\left(c^{\prime}\right), \sum_{h^{\prime}=1}^{H[c]} v_{h^{\prime}}, e[c], H[c]\right)=\max U^{\theta *}\left[c, \theta^{\prime}\right]
$$

must hold for all $\theta \in \Theta\left[c^{\prime}\right]$. Now, suppose that $m^{\epsilon s *}\left(c^{\prime}\right)=0$. Then, $\left(x^{\theta \epsilon s *}\left(c^{\prime}\right), v^{\theta \epsilon s *}\left(c^{\prime}\right)\right)_{\theta \in \Theta\left[c^{\prime}\right]}$ achieves intraclub Pareto efficiency. Since $\left(x^{\theta}\left(c^{\prime}\right), v^{\theta}\left(c^{\prime}\right)\right)_{\theta \in \Theta\left[c^{\prime}\right]}$ is feasible, (i) at least one type $\theta$ strictly prefers $\left(x^{\theta \epsilon s *}\left(c^{\prime}\right), v^{\theta \epsilon s *}\left(c^{\prime}\right)\right)_{\theta \in \Theta\left[c^{\prime}\right]}$ to $\left(x^{\theta}\left(c^{\prime}\right), v^{\theta}\left(c^{\prime}\right)\right)_{\theta \in \Theta\left[c^{\prime}\right]}$, or (ii) all types in $\Theta\left[c^{\prime}\right]$ weakly prefer the former to the latter. In the former case, $\theta$ would choose $c^{\prime}$ in mapping $\rho$, which is a contradiction. In the latter case, if all types are indifferent between $\left(x^{\theta \epsilon s *}\left(c^{\prime}\right), v^{\theta \epsilon s *}\left(c^{\prime}\right)\right)_{\theta \in \Theta\left[c^{\prime}\right]}$ and $\left(x^{\theta}\left(c^{\prime}\right), v^{\theta}\left(c^{\prime}\right)\right)_{\theta \in \Theta\left[c^{\prime}\right.}$, then there is no contradiction, but this implies that $\left(x^{\theta}\left(c^{\prime}\right), v^{\theta}\left(c^{\prime}\right)\right)_{\theta \in \Theta\left[c^{\prime}\right]}$ achieves intraclub Pareto efficiency. We have derived the desired conclusion.

The rest of the proof is straightforward. Mapping $\chi$ assures that each (potential) club manager for each $c \in \mathcal{C}$ chooses the cost-minimizing input vector (Condition 1). Mapping $\rho$ assures that all types of consumers are choosing their most favorite policies (including policies catering to other types of consumers) at the favorite clubs (Condition 6). Mapping $\mu$ shows that only profit-maximizing club-types are provided in the fixed point (and zero profit is assured by the free entry condition: Conditions 2 and 3). Finally, with the Walras law, mappings $\zeta$ and $\sigma$ guarantee that there is no excess demand in commodities and club policies, $z^{\epsilon s *} \leq 0$, in the fixed point by the GaleNikaido Lemma (see Debreu 1959, 5.6). Thus, Conditions 7 and 8 are met (Condition 8 with possible inequalities by truncations). Hence, we conclude that the fixed point of $\varphi$ achieves a social equilibrium for an $(\epsilon, s)$-truncated economy.

Now, let us take $\epsilon$ to zero. Since in the fixed point, each club earns zero profit, if $\lim _{\epsilon \rightarrow 0} p^{\epsilon s}=0$ then $\lim _{\epsilon \rightarrow 0} q^{\epsilon s}=0$. Since $\Delta$ is a simplex, this is a contradiction. Thus, $\lim _{\epsilon \rightarrow 0} p^{\epsilon s *}=p^{s *}=0$ does not occur. Let the limit of convergent sequence when $\epsilon$ goes to zero be $\left(x^{s *}, v^{s *}, I^{s *}, y^{s *}, r^{s *}, m^{s *}, z_{p}^{s *}, z_{r}^{s *}, p^{s *}, q^{s *}\right)$. 
By continuity, this fixed point is still a social equilibrium for an $s$-truncated economy. Now, we take $s$ to infinity following Aumann (1966). In this case, in some $c \in \mathcal{C}$ and $\theta \in \Theta$, the consumption vector may grow unboundedly as $s$ goes to infinity (it can happen if the population measure of $c$ goes to zero, and price goes to zero). Thus, we will work on the limit of the aggregated consumption vector. Let $\left(\bar{x}^{s *}(c), \bar{y}^{s *}(c)\right) \equiv\left(\left(m^{s *}(c) n^{\theta}[c] x^{\theta s *}(c)\right)_{\theta \in \Theta[c]}, m^{s *}(c) y^{s *}(c)\right)$. Consider a sequence $\left\{\left(\bar{x}^{s *}, v^{s *}, I^{s *}, \bar{y}^{s *}, r^{s *}, m^{s *}, z_{p}^{s *}, z_{r}^{s *}, p^{s *}, q^{s *}\right)\right\}_{s=\underline{s}}^{\infty}$. Since $\bar{x}^{s *}(c), \bar{y}^{s *}(c) \leq \sum_{\theta \in \Theta} N^{\theta} \omega^{\theta}$ follows in the fixed points, the sequence lies in a compact set, and there is a convergent sequence. Let the limit of the equilibrium sequence be $\left(\bar{x}^{*}, v^{*}, I^{*}, \bar{y}^{*}, r^{*}, m^{*}, z_{p}^{*}, z_{r}^{*}, p^{*}, q^{*}\right)$. Since preferences are strictly monotonic, $p_{\ell}^{*}>0$ holds for all $\ell=1, \ldots, L$. For clubs with $m^{*}(c)>0$, let $x^{\theta *}(c)=\bar{x}^{\theta *}(c) / m^{*}(c) n^{\theta}[c]$ for all $\theta$, and for clubs with $m^{*}(c)=0$, let $x^{\theta *}(c)=\arg \max _{x \in \mathbb{R}_{+}^{L}} u^{\theta}\left(x, v^{\theta *}, \sum_{\theta \in \Theta[c]} n^{\theta}[c] v^{\theta *}(c), e[c], H[c]\right)$ subject to $p^{*} x \leq p^{*} \omega^{\theta}-q^{\theta}(c)$. Let $y^{*}(c) \in \arg \min p^{*} Y[e[c]]$. Since $p_{\ell}^{*}>0$ for all $\ell=1, \ldots, L$, everything is well defined. By the continuity of utility function, $\left(x^{*}, v^{*}, I^{*}, y^{*}, r^{*}, m^{*}, z_{p}^{*}, z_{r}^{*}, p^{*}, q^{*}\right)$ satisfies all equilibrium conditions. Thus, there exists a social equilibrium in this economy. This completes the proof of Proposition 6.

Now, let us return to the entrepreneurial equilibrium. The main difference between the two equilibrium concepts is that in the entrepreneurial equilibrium, if a club type does not exist, then there is no price and no intraclub allocation for the club type.

Proposition 7. Every social equilibrium is supportable by an entrepreneurial equilibrium, when all fully nonlinear policies are available.

Proof. It is easy to describe type $c \in \mathcal{C}$ club by $\gamma[c] \in \Gamma$ by using policy $q^{\theta}[c](v)$ that satisfies $q^{\theta *}[c](v)=q^{\theta *}(c)$ if $v \leq v^{\theta *}$ and $q^{\theta *}[c](v)=\infty$, otherwise (since preferences are strictly monotonic), with an obvious assignment function $\alpha[c]$. By selecting an arbitrary $\alpha[c]$, we can determine $\gamma[c]$ uniquely for each $c \in \mathcal{C}$. Let $\mathcal{C}^{*}=\left\{\gamma[c] \in \Gamma: m^{*}(c)>0\right\}$. Clearly, $\Gamma^{*}$ is a finite set. Then, since the equilibrium consumption level for $v$ for each seat $h$ is pinned down by a nonlinear policy, no consumer has an incentive to switch from the assigned allocation in social equilibrium (the choice set is smaller, yet includes the equilibrium assignments). Thus, the only condition we need to check is Condition 7 of the entrepreneurial equilibrium.

Suppose that Condition 7 of the entrepreneurial equilibrium is violated. Then, there is a nonexisting club $c^{\prime} \in \mathcal{C} \backslash \mathcal{C}^{*}$ that achieves a positive profit 
and is feasible in the sense that all types of consumers it invites are willing to join. Since the above nonlinear policy system can support any intraclub equilibrium with any policies, we can represent any policy $\gamma^{\prime}$ by club type $c^{\prime}$ with $e\left[c^{\prime}\right],\left(n^{\theta}\left[c^{\prime}\right]\right)_{\theta \in \Theta\left[c^{\prime}\right]}$ and $\left(x^{\theta}\left(c^{\prime}\right), v^{\theta}\left(c^{\prime}\right), q^{\theta}\left[c^{\prime}\right]\right)_{\theta \in \Theta[c]} \in\left(\mathbb{R}_{+}^{L} \times \mathbb{R}_{+}^{K} \times \mathbb{R}_{+}\right)^{\Theta[c]}$ such that

$$
\sum_{\theta \in \Theta\left[c^{\prime}\right]} q^{\theta}\left[c^{\prime}\right] n^{\theta}\left[c^{\prime}\right]-p^{*} Y\left(e\left[c^{\prime}\right]\right)>0
$$

and for all $\theta \in \Theta\left[c^{\prime}\right]$ and all $c \in \mathcal{C}^{*}$ with $n^{\theta}[c]>0$,

$$
\begin{aligned}
& u^{\theta}\left(x^{\theta}\left[c^{\prime}\right], v^{\theta}\left[c^{\prime}\right], \sum_{\theta^{\prime} \in \Theta\left[c^{\prime}\right]} n^{\theta^{\prime}}\left[c^{\prime}\right] v^{\theta}\left[c^{\prime}\right], e\left[c^{\prime}\right], H\left[c^{\prime}\right]\right) \\
> & u^{\theta}\left(x^{\theta *}[c], v^{\theta *}[c], \sum_{\theta^{\prime} \in \Theta\left[c^{\prime}\right]} n^{\theta^{\prime} *}[c] v^{\theta *}[c], e[c], H[c]\right) .
\end{aligned}
$$

Thus, by reducing $q^{\theta}\left[c^{\prime}\right] \mathrm{s}$, there is an intraclub allocation with budget balance that is superior to the social equilibrium allocation. However, since intraclub Pareto-efficiency is achieved in club $c^{\prime}$ in the social equilibrium, there is at least one type who strictly prefers the allocation in club $c^{\prime}$ to the assigned allocation in social equilibrium. Since all types are free to choose their policies in social equilibrium, this cannot happen. This is a contradiction. This completes the proof.

Propositions 5, 6, and 7 prove our Theorem.

\section{Conclusion}

In this paper, we showed that when club members are indivisible and they can choose the intensity of use of facilities, homogeneous clubs may not be efficient even under anonymous crowding effects. We need to allow mixed clubs in order to achieve the first-best allocation. We propose entrepreneurial equilibrium as a solution concept, which is a version of Tiebout equilibrium in Konishi (2008), for mixed clubs with intensity of use of facilities. We show that it exists and is efficient if the available policies for club managers are rich enough. We prove the theorem indirectly: by proving the existence of social equilibrium, which is a core allocation with envy-free property. 
We can generalize our model and theorem. In this paper, we assumed that the input requirement set $Y[e]$ is independent of aggregate intensity of use of facilities $V$. We can relax this assumption by introducing a production set for facility $e \in E: \tilde{Y}[e] \subset \mathbb{R}^{K+L}$ with $(V,-y) \in \tilde{Y}[e]$ being the representative element. If for all $e \in E, \tilde{Y}[e]$ is closed and convex, $\tilde{Y}[e]+\mathbb{R}_{-}^{K+L} \subseteq \tilde{Y}[e]$ (free disposal), $Y[\emptyset]=\mathbb{R}_{-}^{K+L}$ (inaction), and $0 \notin Y[e]$ for all $e \neq \emptyset$ (no free lunch), then we can modify mapping $\delta$ to accommodate this change (see Konishi 2009).

\section{References}

[1] Allouch, N., J.P. Conley, M. Wooders (2009) "Anonymous Price Taking Equilibrium in Tiebout Economies with a Continuum of Agents: Existence and Characterization" Journal of Mathematical Economics 45, pp 492-510.

[2] Aumann, R.J. (1966) "Existence of Competitive Equilibria in Market with a Continuum of Traders" Econometrica 34, pp 1-17.

[3] Berglas, E. (1976) "On the theory of clubs, American Economic Review 66, pp 116-121.

[4] Berglas, E., and D. Pines (1981) "Clubs, Local Public Goods and Transportation Models: a Synthesis" Journal of Public Economics 15, pp 141-162.

[5] Bewley, T.F. (1981) "A Critieque of Tiebout's Theory of Local Public Expenditures" Econometrica 49, pp 713-740.

[6] Boadway, R. (1980) "A Note on the Market Provision of Club Goods" Journal of Public Economics 13, pp 131-137.

[7] Brueckner, J.K., and K. Lee (1991) "Economies of Scope and Multiproduct Clubs" Public Finance Review (Public Finance Quarterly) 19, pp 193-208.

[8] Buchanan, J. (1965) "An Economic Theory of Clubs" Economica 33, pp $1-14$. 
[9] Cornes, R., and T. Sandler (1996) The Theory of Externalities, Public Goods, and Club Goods (Second Edition), New York: Cambridge University Press.

[10] Debreu, G. (1952) "A Social Equilibrium Existence Theorem" Proceedings of National Academy of Science of the USA 38, pp 886-893.

[11] Debreu, G. (1959) Theory of Value, New York: Wiley.

[12] Ellickson, B. (1979) "Competitive Equilibrium with Local Public Goods" Journal of Economic Theory 21, pp 46-61.

[13] Ellickson, B., B. Grodahl, S. Scotchmer, and W.R. Zame (1994) "Clubs and the Market" Econometrica 67, pp 1185-1217.

[14] Foley, D. (1967) "Resource Allocation and the Public Sector" Yale Economic Essays 7, pp 45-98.

[15] Greenberg, J. (1979) "Consistent Majority Rules over Compact Sets of Alternatives" Econometrica 47, pp 627-636.

[16] Hamilton, B.W. (1975) "Zoning and Property Taxation in a System of Local Governments" Urban Studies 12, pp 205-211.

[17] Hart, S., W. Hildenbrand and E. Kohlberg (1974) "On Equilibrium Allocations as Distributions on the Commodity Space" Journal of Mathematical Economics 1, pp 159-166.

[18] Helsley, R.W., and W.C. Strange (1991) "Exclusion and the Theory of Clubs" Canadian Journal of Economics 24, pp 888-899.

[19] Ichiishi, T. (1981) "Social Equilibrium Existence Lemma" Econometrica 49, pp 369-377.

[20] Kaneko, M., and M.H. Wooders (1986) "The Core of a Game with a Continuum of Players and Finite Coalitions: the Model and Some Results" Mathematical Social Sciences 12, pp 105-137.

[21] Konishi, H. (1996) "Voting with Ballots and Feet: Existence of Equilibrium in a Local Public Good Economy" Journal of Economic Theory 68, pp 480-509. 
[22] Konishi, H. (2008) "Tiebout's Tale in Spatial Economies: Entrepreneurship, Self-Selection and Efficiency" Regional Science and Urban Economics 38, pp 461-471, (special issue in honor of Masahisa Fujita).

[23] Konishi, H. (2009) "Housing Market Equilibrium with Entrepreneurial Land Developers: Efficiency of Equilibrium with Local Externalities among Residents (mimeo).

[24] Konishi, H., M. Le Breton, and S. Weber (1997) "Equilibrium in a Model with Partial Rivalry" Journal of Economic Theory 72, pp 225-237.

[25] Mas-Colell, A. (1977) "Indivisible Commodities and General Equilibrium Theory" Journal of Economic Theory 16, pp 443-456.

[26] Mas-Colell, A. (1980) "Efficiency and Decentralization in the Pure Theory of Public Goods" Quarterly Journal of Economics 94, pp 625-641.

[27] Mas-Colell, A. (1984) "On Schmeidler's Theorem" Journal of Mathematical Economics 13, pp 201-206.

[28] Ray, D., and R. Vohra (1997) "Equilibrium Binding Agreement" Journal of Economic Theory 73, pp 30-78.

[29] Rosen, S. (1974) "Hedonic Prices and Implicit Markets: Product Differentiation in Pure Competition" Journal of Political Economy 82, pp $34-55$.

[30] Rothschild, M., and J.E. Stiglitz (1976) "Equilibrium in Competitive Insurance Markets: an Essay on the Economics of Imperfect Information" Quarterly Journal of Economics 40, pp 629-649.

[31] Sandler, T., and J. Tschirhart (1980) "The Economic Theory of Clubs: and Evaluative Survey" Journal of Economic Literature 18, pp 14811521.

[32] Scotchmer, S., "Local public goods and clubs," in A.J. Auerbach and M. Feldstein eds., Handbook of Public Economics Vol.4, Elsevier Science B.V., pp 1997-2042.

[33] Scotchmer, S., and M. Wooders (1987) "Competitive Equilibrium and the Core in Club Economies with Anonymous Crowding" Journal of Public Economics 34, pp 159-173. 
[34] Shafer, W., and H. Sonnenschein (1975) "Equilibrium in Abstract Economies without Ordered Preferences" Journal of Mathematical Economics 2, pp 345-348.

[35] Tiebout, C. (1956) "A Pure Theory of Local Public Expenditures" Journal of Political Economy 64, pp 416-424.

[36] Wooders, M.H. (1978) "Equilibria, the Core, and Jurisdiction Structure in Economies with a Local Public Good" Journal of Economic Theory 18, pp 328-348.

[37] Wooders, M.H. (1999) "Multijurisdictional Economies, the Tiebout Hypothesis, and Sorting" Proceedings of the National Academy of Sciences Vol. 96 (19), pp 10585-10587

[38] Zame, W.R. (2007) "Incentives, Contracts, and Markets: a General Equilibrium Theory of Firms" Econometrica 75, pp 1453-1500. 